\title{
Forward-Secure Group Signatures from Lattices
}

\author{
San Ling, Khoa Nguyen, Huaxiong Wang, Yanhong Xu \\ Division of Mathematical Sciences, \\ School of Physical and Mathematical Sciences, \\ Nanyang Technological University, Singapore. \\ \{lingsan, khoantt, hxwang, xu0014ng\}@ntu.edu.sg
}

\begin{abstract}
Group signature is a fundamental cryptographic primitive, aiming to protect anonymity and ensure accountability of users. It allows group members to anonymously sign messages on behalf of the whole group, while incorporating a tracing mechanism to identify the signer of any suspected signature. Most of the existing group signature schemes, however, do not guarantee security once secret keys are exposed. To reduce potential damages caused by key exposure attacks, Song (ACMCCS 2001) put forward the concept of forward-secure group signature (FSGS), which prevents attackers from forging group signatures pertaining to past time periods even if a secret group signing key is revealed at the current time period. For the time being, however, all known secure FSGS schemes are based on number-theoretic assumptions, and are vulnerable against quantum computers.

In this work, we construct the first lattice-based FSGS scheme. Our scheme is proven secure under the Short Integer Solution and Learning With Errors assumptions. At the heart of our construction is a scalable lattice-based key evolving mechanism, allowing users to periodically update their secret keys and to efficiently prove in zero-knowledge that key evolution process is done correctly. To realize this essential building block, we first employ the Bonsai tree structure by Cash et al. (EUROCRYPT 2010) to handle the key evolution process, and then develop Langlois et al.'s construction (PKC 2014) to design its supporting zeroknowledge protocol.
\end{abstract}

Keywords. Group signatures, key exposure, forward-security, latticebased cryptography, zero-knowledge proofs

\section{Introduction}

Group signatures. Initially suggested by Chaum and van Heyst 20], group signature (GS) allows users of a group controlled by a manager to sign messages anonymously in the name of the group (anonymity). Nevertheless, there is a tracing manager to identify the signer of any signature should the user abuse the anonymity (traceability). These seemingly contractive features, however, allow group signatures to find applications in various real-life scenarios such as e-commence systems and anonymous online communications. Unfortunately, the exposure of group signing keys renders almost all the existing schemes unsatisfactory in practice. Indeed, in the traditional models of group signatures, 
e.g., 6 6 301018/3156, the security of the scheme is no longer guaranteed when the key exposure arises. So now let us look closely at the key exposure problem and the countermeasures to it.

Exposure of Group Signing Keys and Forward-Secure Group SignaTURES. Exposure of users' secret keys is one of the greatest dangers to many cryptographic protocols in practice [57]. Forward-secure mechanisms first introduced by Anderson 4, aim to minimize the damages caused by secret key exposures. More precisely, forward-security protects past uses of private keys in earlier time periods even if a break-in occurs currently. Afterwards, many forward-secure schemes were constructed, such as forward-secure signatures [7/1]27], forwardsecure public key encryption systems 23 179], and forward-secure signatures with un-trusted update 13/41/42. At the heart of these schemes is a key evolving technique that operates as follows. It divides the lifetime of the scheme into discrete $T$ time periods. Upon entering a new time period, a subsequent secret key is computed from the current one via a one-way key evolution algorithm. Meanwhile, the current key is deleted promptly. Due to the one-wayness of the updating algorithm, the security of the previous keys is preserved even though the current one is compromised. Therefore, by carefully choosing a secure scheme that operates well with a key evolving mechanism, forward-security of the scheme can be guaranteed.

As investigated by Song [57, secret key exposure in group signatures is much more damaging than in ordinary digital signatures. In group signatures, if one group member's signing key is disclosed to the attacker, then the latter can sign arbitrary messages. In this situation, if the underlying group signature scheme is not secure against exposure of group signing keys, then the whole system has to be re-initialized, which is obviously inefficient in practice. Besides its inefficiency, this solution is also unsatisfactory. Once there is a break-in of the system, all previously signed group signatures become invalid since we do not have a mechanism to distinguish whether a signature is generated by a legitimate group member or by the attacker. What is worse, one of the easiest way for a misbehaving member Eve to attack the system and/or to repudiate her illegally signed signatures is to reveal her group signing key secretly in the Internet and then claim to be a victim of the key exposure problem [27. Now the users who had accepted signatures before Eve's group signing key is exposed are now at the mercy of all the group members, some of whom (e.g., Eve) would not reissue the signatures with the new key.

The aforementioned problems induced by the exposure of group signing keys motivated Song [57] to put forward the notion of forward-secure group signature (FSGS), in which group members are able to update their group signing keys at each time period via a one-way key evolution algorithm. Therefore, when some group member's singing key is disclosed, all the signatures generated during past periods remain valid, which then prevents dishonest group members from repudiating signatures by simply exposing keys. Later, Nakanishi, Hira, Funabiki [51] defined a rigourous security model of FSGS for static groups, where users are fixed throughout the scheme, and demonstrated a pairing-based construction. 
Subsequently, Libert and Yung [43] extended Nakanishi et al.'s work to capture the setting of the dynamically growing groups. However, all these schemes are constructions based on number-theoretic assumptions and are fragile in the presence of quantum adversaries. In order not to put all eggs in one basket, it is imperative to consider instantiations based on alternative, post-quantum foundations, e.g., lattice assumptions. In view of this, let us now look at the topic of lattice-based group signatures.

Lattice-Based Group signatures. In 2010, Gordon et al. [26] introduced the first lattice-based instantiation of GS. Since then, numerous schemes have been put forward with various improvements on security, efficiency, and functionality. While many of them [16/32 45 $53 / 37 / 1122$ aim to provide enhancement on security and efficiency, they are solely designed for the static groups and often fall too short for specific needs of real-life applications. With regard to advanced features, there have been proposed several schemes [33|34|46 48|40]47] and they are still behind their counterparts in the number-theoretic setting. Specifically, 33 34 46/48 deal with dynamic user enrollments and/or revocations of misbehaving users while [0 47] attempt to restrict the power of the tracing manager or keep his actions accountable. For the time being, the problem of making GS secure against the key exposure problem is still open in the context of lattices. Taking into account the great threat of key exposure to GS and the vulnerability of GS from number-theoretic assumptions in front of quantum computers, it would be tempting to investigate lattice-based instantiations of FSGS. Furthermore, it would be desirable to achieve it with reasonable overhead, e.g., with complexity at most poly-logarithmic in $T$.

Our Contributions. We introduce the first FSGS scheme in the context of lattices. The scheme satisfies the security requirements put forward by Nakanishi et al. 51] in the random oracle model. Assuming the hardness of the Short Integer Solution (SIS) problem and the Learning With Errors (LWE) problem, our scheme achieves full anonymity and a stronger notion of traceability named forward-secure traceability, which captures the traceability in the setting of key exposure problems. Let $\lambda$ be the security parameter, $N$ be the expected number of group members, and $T$ be total time periods, our construction achieves signature size $\widetilde{\mathcal{O}}(\lambda(\log N+\log T))$, group public key size $\widetilde{\mathcal{O}}\left(\lambda^{2}(\log N+\log T)\right)$, and secret key size $\widetilde{\mathcal{O}}\left(\lambda^{2}(\log N+\log T)^{2} \log T\right)$. In particular, forward security is achieved with a reasonable cost: the size of keys and signatures are at most $\mathcal{O}\left(\log ^{3} T\right)$ larger than those of the basic GS scheme [33] upon which we build ours.

Overview of Our Techniques. Typically, designing secure GS requires a combination of digital signature, encryption scheme and zero-knowledge (ZK) protocol. Let us first consider an ordinary GS scheme similar to the template proposed by Bellare et al. [6]. In the scheme, each user is assigned an $\ell$ bit string id as identity, where $\ell=\log N$. The user's signing key is a signature on his identifier id, generated by the group manager. Specifically, we let the signing key be a short vector $\mathbf{v}_{\text {id }}$ satisfying $\mathbf{A}_{\text {id }} \cdot \mathbf{v}_{\text {id }}=\mathbf{u} \bmod q$ for some public vector $\mathbf{u}$. When signing a message, the user first encrypts his identity id to a 
ciphertext $\mathbf{c}$ and proves that he possesses a valid signature on his identity that is also correctly encrypted to c. To achieve forward-security, we would need a mechanism to update the group signing key periodically and a ZK protocol to prove that the key updating procedure is done honestly.

Inspired by the HIBE-like key evolving technique from Nakanishi et al. 51] and Libert and Yung [43, which in turn follows from [17/9/13, we exploit the hierarchical structure of the Bonsai tree [19] to enable periodical key updating. To the best of our knowledge, this is the only lattice-based HIBE in the standard model with supporting (Stern-like [58]) ZK proofs by Langlois et al. [33, which seems to be the right stepping stone towards our goal. Let $T=2^{d}$ be the total number of time periods. To enable key updating, each user id is associated with a subtree of depth $d$, where the leaves of the tree correspond to successive time periods in the apparent way. Let the subtree be identified by matrices $\mathbf{A}_{\mathrm{id}}, \mathbf{A}_{\ell+1}^{0}, \mathbf{A}_{\ell+1}^{1}, \ldots, \mathbf{A}_{\ell+d}^{0}, \mathbf{A}_{\ell+d}^{1}$ and $z=\operatorname{Bin}(t)$ be the binary representation of $t$. In order to show the key evolution is done correctly, we observe that it suffices to prove possession of a (short) Bonsai signature $\mathbf{v}_{\text {id } \| z}$ satisfying $\left[\mathbf{A}_{\mathrm{id}}\left|\mathbf{A}_{\ell+1}^{z[1]}\right| \cdots \mid \mathbf{A}_{\ell+d}^{z[d]}\right] \cdot \mathbf{v}_{\mathrm{id} \| z}=\mathbf{u} \bmod q$. However, proving knowledge of the Bonsai signature departs from the protocol presented in [33]. The matrix $\mathbf{A}_{\text {id }}$ should be secret and the binary string $z$ should be public in our case while it is the other way around in 33 . Nevertheless, analyzing the above equation carefully, it actually reduces to proving knowledge of short vectors $\mathbf{w}_{1}$ and $\mathbf{w}_{2}$ and a binary string id such that $\mathbf{A}_{\mathrm{id}} \cdot \mathbf{w}_{1}+\mathbf{A}^{\prime \prime} \cdot \mathbf{w}_{2}=\mathbf{u} \bmod q$, where $\mathbf{v}_{\mathrm{id} \|}=\left(\mathbf{w}_{1} \| \mathbf{w}_{2}\right)$ and $\mathbf{A}^{\prime \prime}$ is built from some public matrices. To prove knowledge of $\mathbf{w}_{2}$, we can employ the decomposition/extension/permutation techniques by Ling et al. 44] that operate in Stern's framework [58. Regarding the ZK protocol for proving knowledge of $\mathbf{w}_{1}$ and id, it indeed depends on the signature scheme used by the group manager to certify users. For simplicity, we employ the Bonsai tree signature [19] as well. Then, by utilizing the ZK protocol in [33], we are able to prove knowledge of $\mathbf{w}_{1}$ and id and manage to obtain the desired ZK protocol for proving possession of $\mathbf{v}_{\mathrm{id} \| z}$. It is worth mentioning that, besides the Bonsai signature, the Boyen signature [12] is also a plausible candidate, for which a ZK protocol showing the possession of a valid message-signature pair was known [45].

In the above, we have discussed the (Stern-like) ZK protocol showing knowledge of correctly updated signing key $\mathbf{v}_{\mathrm{id} \| \operatorname{Bin}(t)}$, the main technical building block in achieving our FSGS scheme. The next question is then how should the user derive $\mathbf{v}_{\mathbf{i d} \| \operatorname{Bin}(t)}$ for all possible $t$ using his group signing key $\mathbf{v}_{\text {id }}$. To this end, we make a minor but significant change to the group signing key. Observe that for the Bonsai tree signature, once a trapdoor matrix $\mathbf{S}_{\text {id }}$ satisfying $\mathbf{A}_{\text {id }} \cdot \mathbf{S}_{\text {id }}=\mathbf{0} \bmod q$ is known, the user id is able to generate $\mathbf{v}_{\mathrm{id} \| \operatorname{Bin}(t)}$ for all possible $t$. Therefore, we let the user's signing key be $\mathbf{S}_{\text {id }}$ instead. Nevertheless, we then observe user id should not hold $\mathbf{S}_{\mathrm{id}}$ at all times, as the adversary could also generate all possible $\mathbf{v}_{\mathrm{id} \| \operatorname{Bin}(t)}$ once $\mathbf{S}_{\mathrm{id}}$ is known to him. One trivial method is to generate all possible $\mathbf{v}_{\mathrm{id}} \| \operatorname{Bin}(t)$ and then delete all the previous ones upon entering a new period. However, this will incur linear dependency on $T$, which is undesirable for efficiency purpose. 
To achieve logarithmic overhead, we should think of a way to employ the structure of the Bonsai tree. Let $\operatorname{Nodes}_{(t \rightarrow T-1)}$ be the set of nodes such that it has size at most $\log T$ and contains exactly one ancestor of each leaf or the leaf itself between $t$ and $T-1$. Now we let the signing key of user id at time $t$ be trapdoor matrices $\mathbf{S}_{\mathrm{id} \| z}$ for all $z \in \operatorname{Nodes}_{(t \rightarrow T-1)}$. The user is then able to produce all possible $\mathbf{v}_{\mathrm{id} \| \operatorname{Bin}(t)}$ by employing $\mathbf{S}_{\mathrm{id} \| z}$ if $z$ is an ancestor of $\operatorname{Bin}(t)$. More importantly, for each $z^{\prime} \in \operatorname{Nodes}_{(t+1 \rightarrow T-1)}$, there exists a unique ancestor $z \in \operatorname{Nodes}_{(t \rightarrow T-1)}$, which enables the evolving of the signing key from time $t$ to $t+1$, thanks to the basis delegation algorithm of the Bonsai signature.

As discussed so far, we have shown how to update the key periodically and identified the ZK protocol for the honest behaviour of update. The thing that remains is to find a public key encryption (PKE) scheme that is compatible with the above ingredients. Furthermore, to achieve full anonymity, it typically requires the PKE scheme to be CCA-secure. To this end, we apply the CHK transform [18] to the identity-based encryption scheme [25]. For the obtained PKE scheme, we observe that there exists a Stern-like ZK protocol (see [45]) for proving knowledge of the plaintext, which is compatible in our setting.

To summarize, we have obtained a lattice-based FSGS scheme by developing several technical building blocks from previous works in a non-trivial way. Our scheme satisfies full anonymity due to the facts that the underlying encryption scheme is CCA-secure and that the underlying ZK protocol is statistically zeroknowledge, and achieves forward-secure traceability due to the security of the Bonsai tree signature [19]. We believe that, our construction - while not being truly novel - would certainly help to enrich the area of lattice-based GS.

Related Work. Recently, Kansal, Dutta and Mukhopadhyay 28] proposed a lattice-based FSGS scheme that operates in the model of Libert and Yung [43]. Unfortunately, it can be observed that their construction does not satisfy the correctness and security requirements of 43 . (For details, see Appendix A.)

\section{Preliminaries}

Throughout the paper, all vectors are column vectors. When concatenating two matrices of form $\mathbf{A} \in \mathbb{R}^{n \times m}$ and $\mathbf{B} \in \mathbb{R}^{n \times k}$, we use the notion $[\mathbf{A} \mid \mathbf{B}] \in \mathbb{R}^{n \times(m+k)}$ while we denote $(\mathbf{x} \| \mathbf{y}) \in \mathbb{R}^{m+k}$ as the concatenation of two vectors of form $\mathbf{x} \in \mathbb{R}^{m}$ and $\mathbf{y} \in \mathbb{R}^{k}$. Let $[m]$ be the set $\{1,2, \cdots, m\}$.

\subsection{Forward-Secure Group Signatures}

We now recall the syntax and security requirements of forward-secure group signature (FSGS), as formalized by Nakanishi et al. [51]. An FSGS scheme consists of the following polynomial-time algorithms.

${ }^{1}$ This set can be determined by the Nodeselect algorithm presented by Libert and Yung 43. 
KeyGen: This algorithm takes the tuple $(\lambda, T, N)$ as input, with $\lambda$ being security parameter, $T$ being total number of time periods, and $N$ being maximum number of group members. It then returns group public key gpk, secret key msk of group manager (GM), secret key mosk of tracing manager (TM), initial user secret keys $\mathbf{u s k}_{0}$. $\mathbf{u s k}_{0}$ is an array of initial $N$ secret signing key $\left\{\mathbf{u s k}_{0}[0], \mathbf{u s k}_{0}[1], \cdots, \mathbf{u s k}_{0}[N-1]\right\}$, with $\mathbf{u s k}_{0}[i]$ being the initial key of user $i$.

KeyUpdate: On inputs gpk, $\mathbf{u s k}_{t}[i], i$, and $t+1$, with $\mathbf{u s k}_{t}[i]$ being the secret signing key of user $i$ at time $t$, this randomized algorithm outputs the secret signing key $\mathbf{u s k}_{t+1}[i]$ of user $i$ at time $t+1$.

Sign: On inputs gpk, $\mathbf{u s k}_{t}[i]$, user $i$, time period $t$, and message $M$, this randomized algorithm generates a signature $\Sigma$ on message $M$.

Verify: It takes as inputs gpk, time period $t$, message $M$ and signature $\Sigma$, and returns $1 / 0$ indicating the validity of the signature.

Open: On inputs gpk, mosk, $t, M$ and $\Sigma$, this deterministic algorithm returns an index $i$ or $\perp$.

Correctness. For all $\lambda, T, N$, (gpk, msk, mosk, $\left.\mathbf{u s k}_{0}\right) \leftarrow \operatorname{KeyGen}(\lambda, T, N), \forall i \in$

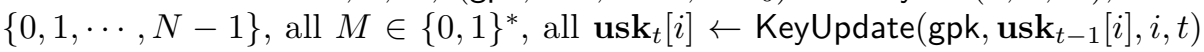
for all $t \in\{0,1, \cdots T-1\}$, the following equations hold:

$$
\begin{gathered}
\operatorname{Verify}\left(\mathrm{gpk}, t, M, \operatorname{Sign}\left(\mathrm{gpk}, \mathbf{u s k}_{t}[i], t, M\right)\right)=1, \\
\text { Open(gpk, mosk, } \left.t, M, \operatorname{Sign}\left(\mathrm{gpk}, \mathbf{u s k}_{t}[i], t, M\right)\right)=i .
\end{gathered}
$$

Forward-Secure Traceability. This requirement demands that any PPT adversary, even if it can corrupt the tracing manager and some (or all) group members, is not able to produce a valid signature (i) that is opened to some non-corrupted user or (ii) that is traced to some corrupted user, but the signature is signed at time period preceding the secret key query of this corrupted user. Note that (i) captures the standard traceability requirement as in [6] while (ii) deals with the new requirement in the context of forward-security. Details are modelled in the experiment in Fig. 1]

In the experiment in Fig. 1, the adversary can adaptively choose which user to corrupt, when to corrupt and when to halt, and when to output its forgery. Furthermore, it is allowed to query signature on any message of a member $i$ through the signing oracle $\operatorname{Sign}\left(\mathbf{u s k}_{t}[\cdot], \cdot\right)$ if $i \notin \mathrm{CU}$ at time period $t$.

Define the advantage $\operatorname{Adv}_{\mathrm{FSGS}, \mathcal{A}}^{\text {Trace }}(\lambda, T, N)$ of adversary $\mathcal{A}$ against forwardsecure traceability of an FSGS scheme as $\operatorname{Pr}\left[\operatorname{Exp}_{\mathrm{FSGS}, \mathcal{A}}^{\text {Trace }}(\lambda, T, N)=1\right]$. An FSGS scheme is forward-secure traceable if the advantage of any PPT adversary is negligible.

Full Anonymity. This requirement demands that any PPT adversary is infeasible to figure out which of two signers of its choice signed the challenged message of its choice at time period $t$ of its choice. Details of this requirement is modelled in the experiment in Fig. 2, In the experiment in Fig. 2, the adversary is accessible to secret keys of all group users and GM, and can query the opening of any signature except for the challenged one through the opening oracle Open(mosk, $\cdot)$. 


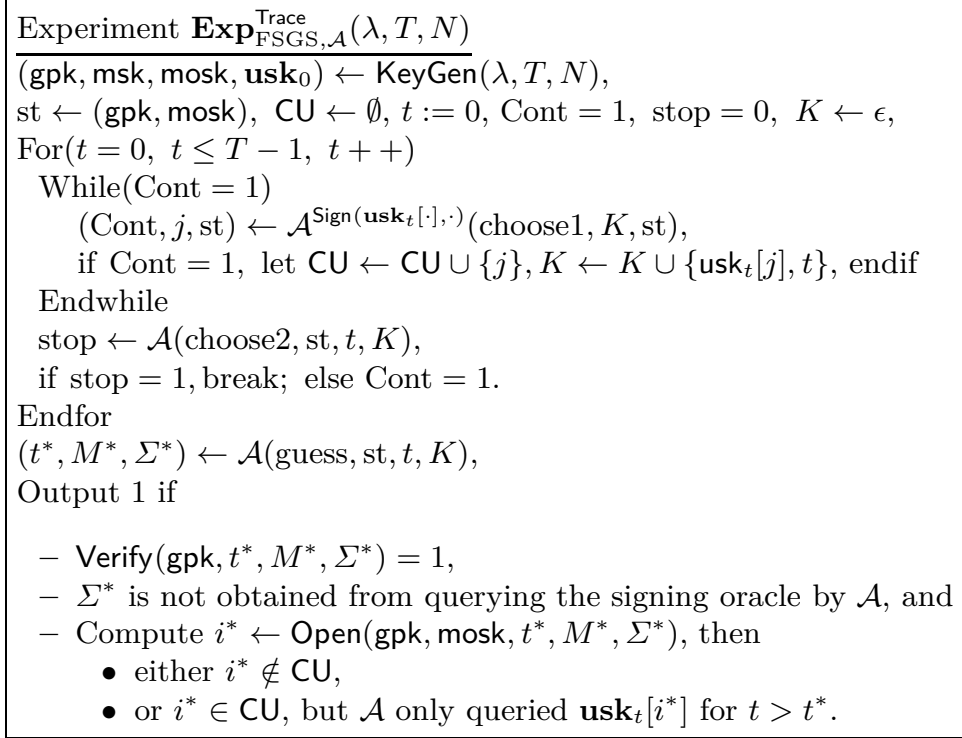

Fig. 1: Experiment used to define forward-secure traceability of an FSGS scheme.

Define $\operatorname{Adv}_{\mathrm{FSGS}, \mathcal{A}}^{\text {Anon }}(\lambda, T, N)$ of $\mathcal{A}$ against full anonymity of an FSGS scheme as $\operatorname{Pr}\left[\operatorname{Exp}_{\mathrm{FSGS}, \mathcal{A}}^{\text {Anon }}(\lambda, T, N)=1\right]$. An FSGS scheme is fully anonymous if the advantage of any PPT adversary $\mathcal{A}$ is negligible.

Experiment $\operatorname{Exp}_{\mathrm{FSGS}, \mathcal{A}}^{\text {Anon }}(\lambda, T, N)$

(gpk, msk, mosk, usk $\left._{0}\right) \leftarrow \operatorname{KeyGen}(\lambda, T, N)$,

(st, $\left.t, i_{0}, i_{1}, M\right) \leftarrow \mathcal{A}^{\text {Open(mosk, } \cdot)}$ (choose, gpk, msk, usk $\left.\mathbf{k}_{0}\right)$,

$b \leftarrow\{0,1\}, \Sigma \leftarrow \operatorname{Sign}\left(\right.$ gpk, usk $\left.{ }_{t}\left[i_{b}\right], t, M\right)$,

$b^{\prime} \leftarrow \mathcal{A}^{\text {Open(mosk, } \cdot)}($ guess, st, $\Sigma)$,

If $b^{\prime}=b$, then output 1 ,

Else output 0.

Fig. 2: Experiment used to define full anonymity of an FSGS scheme.

\subsection{Some Background on Lattices}

Let $n \in \mathbb{Z}^{+}$and $\Lambda$ be a lattice of dimension $n$ over $\mathbb{R}^{n}$. Let $\mathbf{S}=\left\{\mathbf{s}_{1}, \cdots, \mathbf{s}_{n}\right\} \subset$ $\mathbb{R}^{n}$ be a basis of $\Lambda$. For simplicity, we write $\mathbf{S}=\left[\mathbf{s}_{1}|\cdots| \mathbf{s}_{n}\right] \in \mathbb{R}^{n \times n}$. Define $\|\mathbf{S}\|=\operatorname{Max}_{i}\left\|\mathbf{s}_{i}\right\|$. Let $\widetilde{\mathbf{S}}=\left[\widetilde{\mathbf{s}}_{1}|\cdots| \widetilde{\mathbf{s}}_{n}\right]$ be the Gram-Schmidt orthogonalization of S. We refer to $\|\widetilde{\mathbf{S}}\|$ as the Gram-Schmidt norm of $\mathbf{S}$. For any $\mathbf{c} \in \mathbb{R}^{n}$ and $\sigma \in \mathbb{R}^{+}$, define the following: $\rho_{\sigma, \mathbf{c}}(\mathbf{x})=\exp \left(-\pi \frac{\|\mathbf{x}-\mathbf{c}\|^{2}}{\sigma^{2}}\right)$ and $\rho_{\sigma, \mathbf{c}}(\Lambda)=\sum_{\mathbf{x} \in \Lambda} \rho_{\sigma, \mathbf{c}}(\mathbf{x})$ for any $\mathbf{x} \in \Lambda$. Define the discrete Gaussian distribution over the lattice $\Lambda$ with 
parameter $\sigma$ and center $\mathbf{c}$ to be $D_{\Lambda, \sigma, \mathbf{c}}(\mathbf{x})=\rho_{\sigma, \mathbf{c}}(\mathbf{x}) / \rho_{\sigma, \mathbf{c}}(\Lambda)$ for any $\mathbf{x} \in \Lambda$. We often omit $\mathbf{c}$ if it is $\mathbf{0 .}$.

Let $n, m, q \in \mathbb{Z}^{+}$with $q \geq 2$. For $\mathbf{A} \in \mathbb{Z}_{q}^{n \times m}$ and $\mathbf{u} \in \mathbb{Z}_{q}^{n}$ that admits a solution to the equation $\mathbf{A} \cdot \mathbf{x}=\mathbf{u} \bmod q$, define

$$
\Lambda^{\perp}(\mathbf{A})=\left\{\mathbf{e} \in \mathbb{Z}^{m}: \mathbf{A e}=\mathbf{0} \bmod q\right\}, \quad \Lambda^{\mathbf{u}}(\mathbf{A})=\left\{\mathbf{e} \in \mathbb{Z}^{m}: \mathbf{A e}=\mathbf{u} \bmod q\right\} .
$$

Define discrete Gaussian distribution over the set $\Lambda^{\mathbf{u}}(\mathbf{A})$ in the following way: $D_{\Lambda^{\mathbf{u}}(\mathbf{A}), \sigma, \mathbf{c}}(\mathbf{x})=\rho_{\sigma, \mathbf{c}}(\mathbf{x}) / \rho_{\sigma, \mathbf{c}}\left(\Lambda^{\mathbf{u}}(\mathbf{A})\right)$ for $\mathbf{x} \in \Lambda^{\mathbf{u}}(\mathbf{A})$.

Lemma 1 ([25,54]). Let $n, m, q \in \mathbb{Z}^{+}$with $q \geq 2$ and $m \geq 2 n \log q$. Let $\sigma \in \mathbb{R}$ such that $\sigma \geq \omega(\sqrt{\log m})$.

- Then for all but a $2 q^{-n}$ fraction of all $\mathbf{A} \in \mathbb{Z}_{q}^{n \times m}$, the distribution of the syndrome $\mathbf{u}=\mathbf{A} \cdot \mathbf{e} \bmod q$ is within negligible statistical distance from uniform over $\mathbb{Z}_{q}^{n}$ for $\mathbf{e} \hookleftarrow D_{\mathbb{Z}^{m}, \sigma}$. Besides, given $\mathbf{A} \cdot \mathbf{e}=\mathbf{u} \bmod q$, the conditional distribution of $\mathbf{e} \hookleftarrow D_{\mathbb{Z}^{m}, \sigma}$ is $D_{\Lambda^{\mathrm{u}}(\mathbf{A}), \sigma}$.

- Let $x \hookleftarrow D_{\mathbb{Z}, \sigma}, t=\log n$, and $\beta=\lceil\sigma \cdot t\rceil$. Then the probability of $|x| \leq \beta$ is overwhelming.

- The distribution $D_{\mathbb{Z}^{m}, \sigma}$ has min-entropy at least $m-1$.

We next present two hard average-case problems: the Short Integer Solution (SIS) problem (in the $\ell_{\infty}$ norm) and the Learning With Errors (LWE) problem.

Definition $1\left(\left[\begin{array}{l}\mathbf{2}, 5025 \\ \text { 25 }\end{array} \mathrm{SIS}_{n, m, q, \beta}^{\infty}\right)\right.$. Given $\mathbf{A} \stackrel{\$}{\leftarrow} \mathbb{Z}_{q}^{n \times m}$, find a vector $\mathbf{e} \in \mathbb{Z}^{m}$ so that $\mathbf{A} \cdot \mathbf{e}=\mathbf{0} \bmod q$ and $0<\|\mathbf{e}\|_{\infty} \leq \beta$.

Let $q>\beta \sqrt{n}$ be an integer and $m, \beta$ be polynomials in $n$, then solving the $\mathrm{SIS}_{n, m, q, \beta}^{\infty}$ problem (in the $\ell_{\infty}$ norm) is no easier than solving the SIVP $\mathrm{P}_{\gamma}$ problem in the worst-case for some $\gamma=\beta \cdot \widetilde{\mathcal{O}}(\sqrt{n m})$ (see [25[49]).

Definition 2 ([55], $\left.\operatorname{LWE}_{n, q, \chi}\right)$. For $\mathbf{s} \in \mathbb{Z}_{q}^{n}$, define a distribution $\mathcal{A}_{\mathbf{s}, \chi}$ over $\mathbb{Z}_{q}^{n} \times \mathbb{Z}_{q}$ as follows: it samples a uniform vector a over $\mathbb{Z}_{q}^{n}$ and an element $e$ according to $\chi$, and outputs the pair $\left(\mathbf{a}, \mathbf{a}^{\top} \cdot \mathbf{s}+e\right)$. Then the goal of the $\mathrm{LWE}_{n, q, \chi}$ problem is to distinguish $m=\operatorname{poly}(n)$ samples chosen according to the distribution $\mathcal{A}_{\mathbf{s}, \chi}$ for some secret $\mathbf{s} \in \mathbb{Z}_{q}^{n}$ from $m$ samples chosen according to the uniform distribution over $\mathbb{Z}_{q}^{n} \times \mathbb{Z}_{q}$.

Let $B=\widetilde{\mathcal{O}}(\sqrt{n})$ and $\chi$ be an efficiently samplable distribution over $\mathbb{Z}$ that outputs samples $e \in \mathbb{Z}$ with $|e| \leq B$ with all but negligible probability in $n$. If $q \geq 2$ is an arbitrary modulus, then the $\operatorname{LWE}_{n, q, \chi}$ problem is at least as hard as the worst-case problem $\mathrm{SIVP}_{\gamma}$ with $\gamma=\widetilde{\mathcal{O}}(n \cdot q / B)$ through an efficient quantum reduction [55]14]. Additionally, it is showed that the hardness of the LWE problem is maintained when the secret $\mathbf{s}$ is chosen from the error distribution $\chi$ (see [5]).

Now let us recall some algorithms from previous works that will be used extensively in this work.

Lemma 2 ([3]). Let $n, m, q \in \mathbb{Z}^{+}$with $q \geq 2$ and $m=O(n \log q)$. There is a PPT algorithm TrapGen $(n, m, q)$ which returns a tuple $(\mathbf{A}, \mathbf{S})$ such that 
- A is within negligible statistical distance from uniform over $\mathbb{Z}_{q}^{n \times m}$,

- $\mathbf{S}$ is a basis for $\Lambda^{\perp}(\mathbf{A})$, i.e., $\mathbf{A} \cdot \mathbf{S}=0 \bmod q$, and $\|\widetilde{\mathbf{S}}\| \leq \mathcal{O}(\sqrt{n \log q})$.

Lemma $3([\mathbf{2 5}])$. Let $\mathbf{S} \in \mathbb{Z}^{m \times m}$ be a basis of $\Lambda^{\perp}(\mathbf{A})$ for some $\mathbf{A} \in \mathbb{Z}_{q}^{n \times m}$ whose columns expand the entire group $\mathbb{Z}_{q}^{n}$. Let $\mathbf{u}$ be a vector over $\mathbb{Z}_{q}^{n}$ and $s \geq$ $\omega(\sqrt{\log n}) \cdot\|\widetilde{\mathbf{S}}\|$. There is a PPT algorithm $\operatorname{SampleD}(\mathbf{A}, \mathbf{S}, \mathbf{u}, s)$ which returns a vector $\mathbf{v} \in \Lambda^{\mathbf{u}}(\mathbf{A})$ from a distribution that is within negligible statistical distance from $D_{\Lambda^{\mathrm{u}}(\mathbf{A}), s}$.

We also need the following two algorithms to securely delegate basis.

Lemma 4 ([19]). Let $\mathbf{S} \in \mathbb{Z}^{m \times m}$ be a basis of $\Lambda^{\perp}(\mathbf{A})$ for some $\mathbf{A} \in \mathbb{Z}_{q}^{n \times m}$ whose columns generate the entire group $\mathbb{Z}_{q}^{n}$. Let $\mathbf{A}^{\prime} \in \mathbb{Z}_{q}^{n \times m^{\prime}}$ be any matrix containing $\mathbf{A}$ as a submatrix. There is a deterministic polynomial-time algorithm $\operatorname{ExtBasis}\left(\mathbf{S}, \mathbf{A}^{\prime}\right)$ which returns a basis $\mathbf{S}^{\prime} \in \mathbb{Z}^{m^{\prime} \times m^{\prime}}$ of $\Lambda^{\perp}\left(\mathbf{A}^{\prime}\right)$ with $\left\|\widetilde{\mathbf{S}^{\prime}}\right\|=\|\widetilde{\mathbf{S}}\|$.

Lemma 5 ([19]). Let $\mathbf{S}$ be a basis of an $m$-dimensional integer lattice $\Lambda$ and a parameter $s \geq \omega(\sqrt{\log n}) \cdot\|\widetilde{\mathbf{S}}\|$. There is a $\mathrm{PPT}$ algorithm $\operatorname{RandBasis}(\mathbf{S}, s)$ that outputs a new basis $\mathbf{S}^{\prime}$ of $\Lambda$ with $\left\|\mathbf{S}^{\prime}\right\| \leq s \cdot \sqrt{m}$. Moreover, for any two bases $\mathbf{S}_{0}, \mathbf{S}_{1}$ of $\Lambda$ and any $s \geq \max \left\{\left\|\widetilde{\mathbf{S}_{0}}\right\|,\left\|\widetilde{\mathbf{S}_{1}}\right\|\right\} \cdot \omega(\sqrt{\log n})$, the outputs of $\operatorname{RandBasis}\left(\mathbf{S}_{0}, s\right)$ and $\operatorname{RandBasis}\left(\mathbf{S}_{1}, s\right)$ are statistically close.

\subsection{The Bonsai Tree Signature Scheme}

Our construction builds on the Bonsai tree signature scheme [19]. Now we describe it briefly. The scheme takes the following parameters: $\lambda$ is the security parameter and $n=\mathcal{O}(\lambda), \ell$ is the message length, integer $q=\operatorname{poly}(n)$ is sufficiently large, $m=\mathcal{O}(n \log q), \widetilde{L}=\mathcal{O}(\sqrt{n \log q}), s=\omega(\sqrt{\log n}) \cdot \widetilde{L}$, and $\beta=\lceil s \cdot \log n\rceil$. The verification key is the tuple $\left(\mathbf{A}_{0}, \mathbf{A}_{1}^{0}, \mathbf{A}_{1}^{1}, \ldots, \mathbf{A}_{\ell}^{0}, \mathbf{A}_{\ell}^{1}, \mathbf{u}\right)$ while the signing key is $\mathbf{S}_{0}$, where $\left(\mathbf{A}_{0}, \mathbf{S}_{0}\right)$ is generated by the $\operatorname{TrapGen}(n, m, q)$ algorithm as described in Lemma 2 and matrices $\mathbf{A}_{1}^{0}, \mathbf{A}_{1}^{1}, \ldots, \mathbf{A}_{\ell}^{0}, \mathbf{A}_{\ell}^{1}$ and vector $\mathbf{u}$ are all uniformly random and independent over $\mathbb{Z}_{q}^{n \times m}$ and $\mathbb{Z}_{q}^{n}$, respectively.

To sign a binary message id $\in\{0,1\}^{\ell}$, the signer first computes the ma$\operatorname{trix} \mathbf{A}_{\mathrm{id}}:=\left[\mathbf{A}_{0}\left|\mathbf{A}_{1}^{\mathrm{id}[1]}\right| \cdots \mid \mathbf{A}_{\ell}^{\mathrm{id}[\ell]}\right] \in \mathbb{Z}_{q}^{n \times(\ell+1) m}$, and then outputs a vector $\mathbf{v} \in$ $\Lambda^{\mathbf{u}}\left(\mathbf{A}_{\text {id }}\right)$ via the algorithm SampleD(ExtBasis $\left.\left(\mathbf{S}_{0}, \mathbf{A}_{\text {id }}\right), \mathbf{u}, s\right)$. To verify the validity of $\mathbf{v}$ on message id, the verifier computes $\mathbf{A}_{\mathrm{id}}$ as above and checks if $\mathbf{A}_{\mathrm{id}} \cdot \mathbf{v}=\mathbf{u}$ $\bmod q$ and $\|\mathbf{v}\|_{\infty} \leq \beta$ hold. They proved that this signature scheme is existential unforgeable under static chosen message attacks based on the hardness of the SIS problem.

\subsection{Stern-Like Zero-Knowledge Argument Systems}

The statistical zero-knowledge argument of knowledge (ZKAoK) presented in this work are Stern-like [58] protocols. In 1996, Stern [58] suggested a three-move zero-knowledge protocol for the well-known syndrome decoding (SD) problem. 
It was then later adapted to the lattice setting for a restricted version of Inhomogeneous Short Integer Solution (ISIS ${ }^{\infty}$ ) problem by Kawachi et al. 29. More recently, Ling et al. 44] generalized the protocol to handle more versatile relations that find applications in the designs of various lattice-based constructions (see, e.g., 37|35|52 38|36|39]). Libert et al. 34] put forward an abstraction of Stern's protocol to capture a wider range of lattice-based relations, which we now recall.

An abstraction of Stern's Protocol. Let $K, L, q \in \mathbb{Z}^{+}$with $L \geq K$ and $q \geq 2$, and let VALID $\subset\{-1,0,1\}^{L}$. Given a finite set $\mathcal{S}$, associate every $\phi \in \mathcal{S}$ with a permutation $\Gamma_{\phi}$ of $L$ elements so that the following conditions hold:

$$
\left\{\begin{array}{l}
\mathbf{w} \in \mathrm{VALID} \Longleftrightarrow \Gamma_{\phi}(\mathbf{w}) \in \mathrm{VALID}, \\
\text { If } \mathbf{w} \in \mathrm{VALID} \text { and } \phi \text { is uniform in } \mathcal{S}, \text { then } \Gamma_{\phi}(\mathbf{w}) \text { is uniform in VALID. }
\end{array}\right.
$$

The target is to construct a statistical ZKAoK for the abstract relation of the following form:

$$
\mathrm{R}_{\text {abstract }}=\left\{(\mathbf{M}, \mathbf{u}), \mathbf{w} \in \mathbb{Z}_{q}^{K \times L} \times \mathbb{Z}_{q}^{K} \times \mathrm{VALID}: \mathbf{M} \cdot \mathbf{w}=\mathbf{u} \bmod q \cdot\right\}
$$

To obtain the desired ZKAoK protocol, one has to prove that $\mathbf{w} \in$ VALID and $\mathbf{w}$ satisfies the linear equation $\mathbf{M} \cdot \mathbf{w}=\mathbf{u} \bmod q$. To prove the former condition holds in a ZK manner, the prover chooses $\phi \stackrel{\$}{S}$ and let the verifier check $\Gamma_{\phi}(\mathbf{w}) \in$ VALID. According to the first condition in (1), the verifier should be convinced that $\mathbf{w}$ is indeed from the set VALID. At the same time, the verifier is not able to learn any extra information about $\mathbf{w}$ due to the second condition in (11). To show in ZK that the linear equation holds, the prover simply chooses $\mathbf{r}_{w} \stackrel{\$}{\leftarrow} \mathbb{Z}_{q}^{L}$ as a masking vector and then shows to the verifier that the equation $\mathbf{M} \cdot\left(\mathbf{w}+\mathbf{r}_{w}\right)=\mathbf{M} \cdot \mathbf{r}_{w}+\mathbf{u} \bmod q$ holds instead.

Figure 3 describes the interaction between the prover $\mathcal{P}$ and the verifier $\mathcal{V}$ in details. The system utilizes a statistically hiding and computationally binding string commitment scheme COM from [29].

Theorem 1 ([34]). Let COM be a statistically hiding and computationally binding string commitment scheme. Then the interactive depicted in Figure 3 is a statistical ZKAoK with perfect completeness, soundness error $2 / 3$, and communication $\operatorname{cost} \mathcal{O}(L \log q)$. Specifically:

- There exists a polynomial-time simulator that, on input $(\mathbf{M}, \mathbf{u})$, with probability $2 / 3$ it outputs an accepted transcript that is within negligible statistical distance from the one produced by an honest prover who knows the witness.

- There is an algorithm that, takes as inputs $(\mathbf{M}, \mathbf{u})$ and three accepting transcripts $\left(\mathrm{CMT}, 1, \mathrm{RSP}_{1}\right),\left(\mathrm{CMT}, 2, \mathrm{RSP}_{2}\right),\left(\mathrm{CMT}, 3, \mathrm{RSP}_{3}\right)$ on $(\mathbf{M}, \mathbf{u})$, and outputs $\mathbf{w}^{\prime} \in$ VALID such that $\mathbf{M} \cdot \mathbf{w}^{\prime}=\mathbf{u} \bmod q$ in polynomial time.

The proof of the Theorem 1, appeared in [34, is omitted here. 
1. Commitment: Prover chooses $\mathbf{r}_{w} \stackrel{\$}{\leftarrow} \mathbb{Z}_{q}^{L}, \phi \stackrel{\$}{\leftarrow} \mathcal{S}$ and randomness $\rho_{1}, \rho_{2}, \rho_{3}$ for COM. Then he sends CMT $=\left(C_{1}, C_{2}, C_{3}\right)$ to the verifier, where

$$
\begin{gathered}
C_{1}=\operatorname{CoM}\left(\phi, \mathbf{M} \cdot \mathbf{r}_{w} \bmod q ; \rho_{1}\right), \quad C_{2}=\operatorname{CoM}\left(\Gamma_{\phi}\left(\mathbf{r}_{w}\right) ; \rho_{2}\right), \\
C_{3}=\operatorname{CoM}\left(\Gamma_{\phi}\left(\mathbf{w}+\mathbf{r}_{w} \bmod q\right) ; \rho_{3}\right) .
\end{gathered}
$$

2. Challenge: $\mathcal{V}$ randomly choose a challenge $C h$ from the set $\{1,2,3\}$ and sends it to $\mathcal{P}$.

3. Response: According to the choice of $C h, \mathcal{P}$ sends back response RSP computed in the following manner:

$-C h=1$ : Let $\mathbf{t}_{w}=\Gamma_{\phi}(\mathbf{w}), \mathbf{t}_{r}=\Gamma_{\phi}\left(\mathbf{r}_{w}\right)$, and RSP $=\left(\mathbf{t}_{w}, \mathbf{t}_{r}, \rho_{2}, \rho_{3}\right)$.

$-C h=2$ : Let $\phi_{2}=\phi, \mathbf{w}_{2}=\mathbf{w}+\mathbf{r}_{w} \bmod q$, and $\operatorname{RSP}=\left(\phi_{2}, \mathbf{w}_{2}, \rho_{1}, \rho_{3}\right)$.

$-C h=3$ : Let $\phi_{3}=\phi, \mathbf{w}_{3}=\mathbf{r}_{w}$, and $\operatorname{RSP}=\left(\phi_{3}, \mathbf{w}_{3}, \rho_{1}, \rho_{2}\right)$.

Verification: When receiving $\operatorname{RSP}$ from $\mathcal{P}, \mathcal{V}$ performs as follows:

$-C h=1$ : Check that $\mathbf{t}_{w} \in \operatorname{VALID}, C_{2}=\operatorname{COM}\left(\mathbf{t}_{r} ; \rho_{2}\right), C_{3}=\operatorname{COM}\left(\mathbf{t}_{w}+\mathbf{t}_{r} \bmod q ; \rho_{3}\right)$.

$-C h=2:$ Check that $C_{1}=\operatorname{COM}\left(\phi_{2}, \mathbf{M} \cdot \mathbf{w}_{2}-\mathbf{u} \bmod q ; \rho_{1}\right), C_{3}=\operatorname{COM}\left(\Gamma_{\phi_{2}}\left(\mathbf{w}_{2}\right) ; \rho_{3}\right)$.

$-C h=3:$ Check that $C_{1}=\operatorname{COM}\left(\phi_{3}, \mathbf{M} \cdot \mathbf{w}_{3} ; \rho_{1}\right), C_{2}=\operatorname{COM}\left(\Gamma_{\phi_{3}}\left(\mathbf{w}_{3}\right) ; \rho_{2}\right)$.

In each case, $\mathcal{V}$ returns 1 if and only if all the conditions hold.

Fig. 3: Stern-like ZKAoK for the relation $\mathrm{R}_{\text {abstract }}$. 


\section{Our Lattice-Based Forward-Secure Group Signature}

In the description below, for a binary tree of depth $k$, we identify each node at depth $j$ with a binary vector $z$ of length $j$ such that $z[1]$ to $z[j]$ are ordered from the top to the bottom and a 0 and a 1 indicate the left and right branch respectively in the order of traversal. Let $B \in \mathbb{Z}^{+}$. For an integer $0 \leq b \leq B$, denote $\operatorname{Bin}(b)$ as the binary representation of $b$ with length $\lceil\log B\rceil$.

In our FSGS scheme, lifetime of the scheme is divided into $T$ discrete periods $0,1, \cdots, T-1$. For simplicity, let $T=2^{d}$ for some $d \in \mathbb{Z}^{+}$. Following previous works [13 43, each time period $t$ is associated with leaf $\operatorname{Bin}(t)$.

Following [13], for $j=1, \cdots, d+1, t \in\{0,1, \cdots, T-1\}$, we define a time period's "right sibling at depth $j$ " as

$$
\operatorname{sibling}(j, t)= \begin{cases}(1)^{\top} & \text { if } j=1 \text { and } \operatorname{Bin}(t)[j]=0, \\ (\operatorname{Bin}(t)[1], \cdots, \operatorname{Bin}(t)[j-1], 1)^{\top} & \text { if } 1<j \leq d \text { and } \operatorname{Bin}(t)[j]=0, \\ \perp & \text { if } j \leq d \text { and } \operatorname{Bin}(t)[j]=1, \\ \operatorname{Bin}(t) & \text { if } j=d+1 .\end{cases}
$$

Define node set $\operatorname{Nodes}_{(t \rightarrow T-1)}$ to be $\{\operatorname{sibling}(1, t), \cdots, \operatorname{sibling}(d+1, t)\}$. For any $t^{\prime}>t$, one can check that for any non- $\perp z^{\prime} \in \operatorname{Nodes}_{\left(t^{\prime} \rightarrow T-1\right)}$, there exists a $z \in \operatorname{Nodes}_{(t \rightarrow T-1)}$ such that $z$ is an ancestor of $z^{\prime}$.

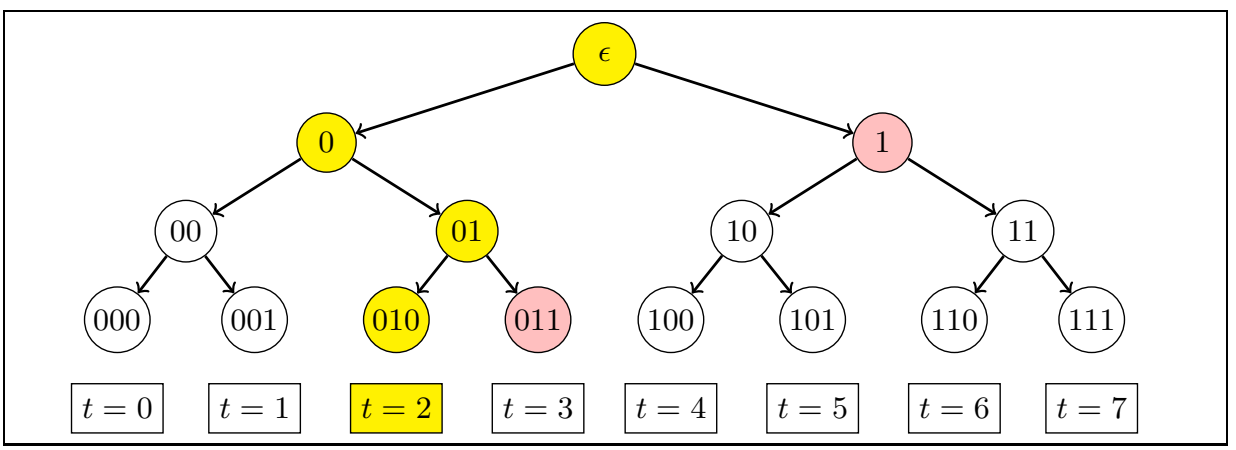

Fig. 4: A binary tree with time periods $T=2^{3}$. Consider the path from the root $\epsilon$ to the leaf node $(010)^{\top}$, we have $\operatorname{sibling}(1,2)=(1)^{\top}$, sibling $(2,2)=\perp$, $\operatorname{sibling}(3,2)=(011)^{\top}$, and sibling $(4,2)=(010)^{\top}$. Therefore, $\operatorname{Nodes}_{(2 \rightarrow 7)}=$ $\left\{(1)^{\top}, \perp,(011)^{\top},(010)^{\top}\right\}$. Similarly, $\operatorname{Nodes}_{(5 \rightarrow 7)}=\left\{\perp,(11)^{\top}, \perp,(101)^{\top}\right\}$. It is verifiable that node $(1)^{\top}$ is an ancestor of both node $(101)^{\top}$ and node $(11)^{\top}$.

\subsection{Description of the Scheme}

Our scheme operates in the Nakanishi et al.'s (static) model [51]. Let $T=2^{d}$ and $N=2^{\ell}$. The group public key consists of (i) a Bonsai tree of depth $\ell+d$ 
specified by a matrix $\mathbf{A}=\left[\mathbf{A}_{0}\left|\mathbf{A}_{1}^{0}\right| \mathbf{A}_{1}^{1} \cdots\left|\mathbf{A}_{\ell+d}^{0}\right| \mathbf{A}_{\ell+d}^{1}\right] \in \mathbb{Z}_{q}^{n \times(2 \ell+2 d+1) m}$ and a vector $\mathbf{u} \in \mathbb{Z}_{q}^{n}$, which are for issuing certificate; (ii) A public matrix $\mathbf{B} \in \mathbb{Z}_{q}^{n \times m}$ of the IBE scheme by Gentry et al. [25], which is for encrypting user identities when signing messages. The secret key of GM is a trapdoor matrix of the Bonsai tree while the secret key of the tracing manager is a trapdoor matrix of the IBE scheme.

Each user id $\in\{0,1\}^{\ell}$ is assigned a node id. To enable periodical key updating, each user id is associated with a subtree of depth $d$. In our scheme, all users are assumed to be valid group members from time 0 to $T-1$. Let $z$ be a binary string of length $d_{z} \leq d$. Define $\mathbf{A}_{\mathrm{id} \| z}=\left[\mathbf{A}_{0}\left|\mathbf{A}_{1}^{\mathrm{id}[1]}\right| \cdots\left|\mathbf{A}_{\ell}^{\mathrm{id}[\ell]}\right| \mathbf{A}_{\ell+1}^{z[1]}|\cdots| \mathbf{A}_{\ell+d_{z}}^{z\left[d_{z}\right]}\right] \in$ $\mathbb{Z}_{q}^{n \times\left(\ell+d_{z}+1\right) m}$. Specifically, the group signing key of user id at time $t$ is $\left\{\mathbf{S}_{\mathrm{id} \| z}, z \in\right.$ $\left.\operatorname{Nodes}_{(t \rightarrow T-1)}\right\}$, which satisfies $\mathbf{A}_{\mathrm{id} \| z} \cdot \mathbf{S}_{\mathrm{id} \| z}=\mathbf{0} \bmod q$. Thanks to the basis delegation technique [19, users are able to compute the trapdoor matrices for all the descendent of nodes in the set $\operatorname{Nodes}_{(t \rightarrow T-1)}$ and hence are able to derive all the subsequent signing keys.We remark that for leaf nodes, it is sufficient to generate short vectors instead of short bases, since we do not need to perform further delegations.

Once received the group signing key, each user can issue signatures on behalf of the group. When signing a message at time $t$, user id first generates a one-time signature key pair (ovk, osk), and then encrypts his identity id to a ciphertext c using the IBE scheme with respect to "identity" ovk. Next, he proves in zero-knowledge that: (i) he is a certified group member; (ii) he has done key evolution honestly; (iii) $\mathbf{c}$ is a correct encryption of id. To prove that facts (i) and (ii) hold, it is sufficient to prove knowledge of a short vector $\mathbf{v}_{\text {id }} \| \operatorname{Bin}(t)$ such that $\mathbf{A}_{\mathrm{id} \| \operatorname{Bin}(t)} \cdot \mathbf{v}_{\mathrm{id} \| \operatorname{Bin}(t)}=\mathbf{u} \bmod q$. The protocol is developed from Langlois et al.'s technique [33] (which was also employed in 21] for designing policy-based signatures) and Ling et al.'s technique [4], and is repeated $\kappa=\omega(\log n)$ times to achieve negligible soundness error, and is made non-interactive via Fiat-Shamir transform [24] as a triple $\Pi$. Finally, the user generates a one-time signature sig on the pair $(\mathbf{c}, \Pi)$, and outputs the group signature consisting of (ovk, $\mathbf{c}, \Pi$, sig).

To verify a group signature, one checks the validity of sig under the key ovk and $\Pi$. In case of dispute, TM can decrypt the ciphertext with respect to the "identity" ovk using his secret key. Details of the scheme are described below.

$\operatorname{KeyGen}(\lambda, T, N)$ : On inputs security parameter $\lambda$, total number of time periods $T=2^{d}$ for some $d \in \mathbb{Z}_{+}$and maximum number of group members $N=2^{\ell}$ for some $\ell \in \mathbb{Z}^{+}$, this algorithm does the following:

1. Choose $n=\mathcal{O}(\lambda), q=\operatorname{poly}(n), m=\mathcal{O}(n \log q)$. Let $k=\ell+d$ and $\kappa=\omega(\log n)$.

2. Run TrapGen $(n, m, q)$ as described in Lemma 2 to obtain $\mathbf{A}_{0} \in \mathbb{Z}_{q}^{n \times m}$ and $\mathbf{S}_{0} \in \mathbb{Z}^{m \times m}$.

3. Sample $\mathbf{u} \stackrel{\$}{\leftarrow} \mathbb{Z}_{q}^{n}$, and $\mathbf{A}_{i}^{b} \stackrel{\$}{\leftarrow} \mathbb{Z}_{q}^{n \times m}$ for all $i \in[k]$ and $b \in\{0,1\}$.

4. Choose a one-time signature scheme $\mathcal{O} \mathcal{T} \mathcal{S}=($ OGen, OSign, OVer $)$, and a statistically hiding and computationally binding commitment scheme COM from [29] that will be used in our zero-knowledge argument system. 
5. Let $\mathcal{H}_{0}:\{0,1\}^{*} \rightarrow \mathbb{Z}_{q}^{n \times \ell}$ and $\mathcal{H}_{1}:\{0,1\}^{*} \rightarrow\{1,2,3\}^{\kappa}$ be collisionresistant hash functions, which will be modelled as random oracles in the security analysis.

6. Let Gaussian parameter $s_{i}$ be $\mathcal{O}(\sqrt{n k \log q})^{i-\ell+1} \cdot \omega(\sqrt{\log n})^{i-\ell+1}$, which will be used to generate short bases or sample short vectors at level $i$ for $i \in\{\ell, \ell+1, \cdots, k\}$.

7. Choose integer bounds $\beta=\left\lceil s_{k} \cdot \log n\right\rceil, B=\widetilde{\mathcal{O}}(\sqrt{n})$, and let $\chi$ be a $B$-bounded distribution over $\mathbb{Z}$.

8. Generate a master key pair $(\mathbf{B}, \mathbf{S}) \in \mathbb{Z}_{q}^{n \times m} \times \mathbb{Z}^{m \times m}$ for the IBE scheme by Gentry et al. 25] via the $\operatorname{TrapGen}(n, m, q)$ algorithm.

9. For user $i \in\{0,1, \cdots, N-1\}$, let id $=\operatorname{Bin}(i) \in\{0,1\}^{\ell}$. Let node id be the identifier of user $i$. Determine the node set $\operatorname{Nodes}_{(0 \rightarrow T-1)}$.

For $z \in \operatorname{Nodes}_{(0 \rightarrow T-1)}$, if $z=\perp$, set $\mathbf{u s k}_{0}[i][z]=\perp$. Otherwise denote $d_{z}$ as the length of $z$ with $d_{z} \leq d$, compute the matrix

$$
\mathbf{A}_{\mathrm{id} \| z}=\left[\mathbf{A}_{0}\left|\mathbf{A}_{1}^{\mathrm{id}[1]}\right| \cdots\left|\mathbf{A}_{\ell}^{\mathrm{id}[\ell]}\right| \mathbf{A}_{\ell+1}^{z[1]}|\cdots| \mathbf{A}_{\ell+d_{z}}^{z\left[d_{z}\right]}\right] \in \mathbb{Z}_{q}^{n \times\left(\ell+d_{z}+1\right) m} .
$$

and proceed as follows:

- If $z$ is of length $d$, i.e., $d_{z}=d$, it computes a vector $\mathbf{v}_{\mathrm{id} \| z} \in \Lambda^{\mathbf{u}}\left(\mathbf{A}_{\mathrm{id} \| z}\right)$ via

$$
\left.\mathbf{v}_{\mathrm{id} \| z} \leftarrow \text { SampleD(ExtBasis }\left(\mathbf{S}_{0}, \mathbf{A}_{\mathrm{id} \| z}\right), \mathbf{u}, s_{k}\right) .
$$

Set $\mathbf{u s k}_{0}[i][z]=\mathbf{v}_{\text {id } \| z}$.

- If $z$ is of length less than $d$, i.e., $1 \leq d_{z}<d$, it computes a matrix $\mathbf{S}_{\mathrm{id} \| z} \in \mathbb{Z}^{\left(\ell+d_{z}+1\right) m \times\left(\ell+d_{z}+1\right) m}$ via

$$
\mathbf{S}_{\mathrm{id} \| z} \leftarrow \operatorname{RandBasis}\left(\operatorname{ExtBasis}\left(\mathbf{S}_{0}, \mathbf{A}_{\mathrm{id} \| z}\right), s_{\ell+d_{z}}\right) .
$$

Set $\mathbf{u s k}_{0}[i][z]=\mathbf{S}_{\mathrm{id} \| z}$.

Let $\mathbf{u s k}_{0}[i]=\left\{\mathbf{u s k}_{0}[i][z], z \in \operatorname{Nodes}_{(0 \rightarrow T-1)}\right\}$ be the initial secret key of user $i$.

Let public parameter be pp, group public key be gpk, secret key of GM be msk, secret key of TM be mosk and initial secret key be $\mathbf{u s k}_{0}$, which are defined as follows:

$$
\begin{gathered}
\mathrm{pp}=\left\{n, q, m, \ell, d, k, \kappa, \mathcal{O} \mathcal{T S}, \mathrm{COM}, \mathcal{H}_{0}, \mathcal{H}_{1}, s_{\ell}, \ldots, s_{k}, \beta, B\right\} \\
\mathrm{gpk}=\left\{\mathrm{pp}, \mathbf{A}_{0}, \mathbf{A}_{1}^{0}, \mathbf{A}_{1}^{1}, \ldots, \mathbf{A}_{k}^{0}, \mathbf{A}_{k}^{1}, \mathbf{u}, \mathbf{B}\right\} \\
\operatorname{msk}=\mathbf{S}_{0}, \quad \operatorname{mosk}=\mathbf{S} \\
\mathbf{u s k}_{0}=\left\{\mathbf{u s k}_{0}[0], \mathbf{u s k}_{0}[1], \ldots, \mathbf{u s k}_{0}[N-1]\right\}
\end{gathered}
$$

KeyUpdate(gpk, $\left.\mathbf{u s k}_{t}[i], i, t+1\right)$ : Compute the identifier of user $i$ as id $=\operatorname{Bin}(i)$, $\operatorname{parse} \mathbf{u s k}_{t}[i]=\left\{\mathbf{u s k}_{t}[i][z], z \in \operatorname{Nodes}_{(t \rightarrow T-1)}\right\}$, and determine the node set $\operatorname{Nodes}_{(t+1 \rightarrow T-1)}$.

For $z^{\prime} \in \operatorname{Nodes}_{(t+1 \rightarrow T-1)}$, if $z^{\prime}=\perp$, set $\mathbf{u s k}_{t+1}[i]\left[z^{\prime}\right]=\perp$. Otherwise, there exists exactly one $z \in \operatorname{Nodes}_{(t \rightarrow T-1)}$ as its prefix, i.e., $z^{\prime}=z \| y$ for some suffix $y$. Consider the following two cases. 
1. If $z^{\prime}=z$, let $\mathbf{u s k}_{t+1}[i]\left[z^{\prime}\right]=\mathbf{u s k}_{t}[i][z]$.

2. If $z^{\prime}=z \| y$ for some non-empty $y$, then $\mathbf{u s k}_{t}[i][z]=\mathbf{S}_{\mathrm{id} \| z}$. Consider the following two subcases.

- If $z^{\prime}$ is of length $d$, run

$$
\mathbf{v}_{\mathrm{id} \| z^{\prime}} \leftarrow \text { SampleD }\left(\text { ExtBasis }\left(\mathbf{S}_{\mathrm{id} \| z}, \mathbf{A}_{\mathrm{id} \| z^{\prime}}\right), \mathbf{u}, s_{k}\right),
$$

and set $\mathbf{u s k}_{t+1}[i]\left[z^{\prime}\right]=\mathbf{v}_{\mathrm{id}} \| z^{\prime}$.

- If $z^{\prime}$ is of length less than $d$, run

$$
\left.\mathbf{S}_{\mathrm{id} \| z^{\prime}} \leftarrow \text { RandBasis(ExtBasis }\left(\mathbf{S}_{\mathrm{id} \| z}, \mathbf{A}_{\mathrm{id} \| z^{\prime}}\right), s_{\ell+d_{z^{\prime}}}\right),
$$

and set $\mathbf{u s k}_{t+1}[i]\left[z^{\prime}\right]=\mathbf{S}_{\mathrm{id} \| z^{\prime}}$.

Output updated key as $\mathbf{u s k}_{t+1}[i]=\left\{\mathbf{u s k}_{t+1}[i]\left[z^{\prime}\right], z^{\prime} \in \operatorname{Nodes}_{(t+1 \rightarrow T-1)}\right\}$.

$\operatorname{Sign}\left(\right.$ gpk, usk $\left._{t}[i], i, t, M\right)$ : Compute the identifier id $=\operatorname{Bin}(i)$. By the structure of the node set $\operatorname{Nodes}_{(t \rightarrow T-1)}$, there exists some $z \in \operatorname{Nodes}_{(t \rightarrow T-1)}$ such that $z=\operatorname{Bin}(t)$ is of length $d$ and $\mathbf{u s k}_{t}[i][z]=\mathbf{v}_{\mathrm{id} \| z}$.

To sign a message $M \in\{0,1\}^{*}$, the signer then performs the following steps.

1. First, generate a one-time signature key pair (ovk, osk) $\leftarrow$ OGen $(n)$, and then encrypt id with respect to "identity" ovk as follows. Let $\mathbf{G}=$ $\mathcal{H}_{0}$ (ovk) $\in \mathbb{Z}_{q}^{n \times \ell}$. Sample $\mathbf{s} \hookleftarrow \chi^{n}, \mathbf{e}_{1} \hookleftarrow \chi^{m}, \mathbf{e}_{2} \hookleftarrow \chi^{\ell}$, and compute ciphertext $\left(\mathbf{c}_{1}, \mathbf{c}_{2}\right) \in \mathbb{Z}_{q}^{m} \times \mathbb{Z}_{q}^{\ell}$ as

$$
\left(\mathbf{c}_{1}=\mathbf{B}^{\top} \cdot \mathbf{s}+\mathbf{e}_{1}, \quad \mathbf{c}_{2}=\mathbf{G}^{\top} \cdot \mathbf{s}+\mathbf{e}_{2}+\left\lfloor\frac{q}{2}\right\rfloor \cdot \mathrm{id}\right) .
$$

2. Second, compute the matrix $\mathbf{A}_{\mathrm{id} \| z}$ and generate a NIZKAoK $\Pi$ to demonstrate the possession of a valid tuple

$$
\xi=\left(\mathrm{id}, \mathbf{s}, \mathbf{e}_{1}, \mathbf{e}_{2}, \mathbf{v}_{\mathrm{id} \| z}\right)
$$

such that

(a) $\mathbf{A}_{\mathrm{id} \| z} \cdot \mathbf{v}_{\mathrm{id} \| z}=\mathbf{u} \bmod q$, and $\left\|\mathbf{v}_{\mathrm{id} \| z}\right\|_{\infty} \leq \beta$.

(b) Equations in (2) hold with $\|\mathbf{s}\|_{\infty} \leq B,\left\|\mathbf{e}_{1}\right\|_{\infty} \leq B$ and $\left\|\mathbf{e}_{2}\right\|_{\infty} \leq B$.

This is done by running our argument system described in Section 4.2 with public input

$$
\gamma=\left(\mathbf{A}_{0}, \mathbf{A}_{1}^{0}, \mathbf{A}_{1}^{1}, \ldots, \mathbf{A}_{k}^{0}, \mathbf{A}_{k}^{1}, \mathbf{u}, \mathbf{B}, \mathbf{G}, \mathbf{c}_{1}, \mathbf{c}_{2}, t\right)
$$

and witness tuple $\xi$ as above. The protocol is repeated $\kappa=\omega(\log n)$ times to obtain negligible soundness error and made non-interactive via the Fiat-Shamir heuristic [24] as a triple $\Pi=\left(\left(\mathrm{CMT}_{i}\right)_{i=1}^{\kappa}, \mathrm{CH},\left(\mathrm{RSP}_{i}\right)_{i=1}^{\kappa}\right)$ with $\mathrm{CH}=\mathcal{H}_{1}\left(M,\left(\mathrm{CMT}_{i}\right)_{i=1}^{\kappa}, \mathbf{c}_{1}, \mathbf{c}_{2}, t\right)$.

3. Third, compute a one-time signature sig $=\operatorname{OSign}\left(\right.$ osk $\left.; \mathbf{c}_{1}, \mathbf{c}_{2}, \Pi\right)$ and output the signature as $\Sigma=$ (ovk, $\mathbf{c}_{1}, \mathbf{c}_{2}, \Pi$, sig).

Verify $(\mathrm{gpk}, t, M, \Sigma)$ : This algorithm proceeds as follows: 
1. Parse $\Sigma$ as $\Sigma=$ (ovk, $\mathbf{c}_{1}, \mathbf{c}_{2}, \Pi$, sig). If OVer(ovk; sig; $\left.\mathbf{c}_{1}, \mathbf{c}_{2}, \Pi\right)=0$, then return 0 .

2. Parse $\Pi$ as $\Pi=\left(\left(\mathrm{CMT}_{i}\right)_{i=1}^{\kappa},\left(\mathrm{Ch}_{1}, \ldots, \mathrm{Ch}_{\kappa}\right),\left(\mathrm{RSP}_{i}\right)_{i=1}^{\kappa}\right)$.

If $\left(\mathrm{Ch}_{1}, \cdots, \mathrm{Ch}_{\kappa}\right) \neq \mathcal{H}_{1}\left(M,\left(\mathrm{CMT}_{i}\right)_{i=1}^{\kappa}, \mathbf{c}_{1}, \mathbf{c}_{2}, t\right)$, then return 0 .

3. For $i \in[\kappa]$, run the verification step of the underlying argument protocol to check the validity of $\mathrm{RSP}_{i}$ with respect to $\mathrm{CMT}_{i}$ and $\mathrm{Ch}_{i}$. If any of the conditions does not hold, then return 0 .

4. Return 1.

Open (gpk, mosk, $t, M, \Sigma)$ : If Verify (gpk, $t, M, \Sigma)=0$, abort. Otherwise, let mosk be $\mathbf{S} \in \mathbb{Z}^{m \times m}$ and parse $\Sigma$ as $\Sigma=$ (ovk, $\mathbf{c}_{1}, \mathbf{c}_{2}, \Pi$, sig). Then it decrypts $\left(\mathbf{c}_{1}, \mathbf{c}_{2}\right)$ as follows:

1. Compute $\mathbf{G}=\mathcal{H}_{0}($ ovk $)=\left[\mathbf{g}_{1}|\cdots| \mathbf{g}_{\ell}\right] \in \mathbb{Z}^{n \times \ell}$. Then use $\mathbf{S}$ to compute a small norm matrix $\mathbf{F}_{\text {ovk }} \in \mathbb{Z}^{m \times \ell}$ such that $\mathbf{B} \cdot \mathbf{F}_{\text {ovk }}=\mathbf{G} \bmod q$. This is done by computing $\mathbf{f}_{i} \leftarrow \operatorname{SampleD}\left(\mathbf{B}, \mathbf{S}, \mathbf{g}_{i}, s_{\ell}\right)$ for all $i \in[\ell]$ and let $\mathbf{F}_{\text {ovk }}=\left[\mathbf{f}_{1}|\cdots| \mathbf{f}_{\ell}\right]$.

2. Use $\mathbf{F}_{\text {ovk }}$ to decrypt $\left(\mathbf{c}_{1}, \mathbf{c}_{2}\right)$ by computing

$$
\operatorname{id}^{\prime}=\left\lfloor\frac{\mathbf{c}_{2}-\mathbf{F}_{\text {ovk }}^{\top} \cdot \mathbf{c}_{1}}{\lfloor q / 2\rfloor}\right\rceil \in\{0,1\}^{\ell} .
$$

3. Return $\operatorname{id}^{\prime} \in\{0,1\}^{\ell}$.

\subsection{Analysis of the Scheme}

EFFICIENCY. We first analyze the complexity of the scheme described in Section 3.1, with respect to security parameter $\lambda$ and parameters $\ell=\log N$ and $d=\log T$. Recall $k=\ell+d$.

- The group public key gpk has bit-size $\widetilde{\mathcal{O}}\left(\lambda^{2} \cdot k\right)$.

- The user secret key $\mathbf{u s k}_{t}[i]$ has at most $d+1$ trapdoor matrices, and has bit-size $\widetilde{\mathcal{O}}\left(\lambda^{2} \cdot k^{2} d\right)$.

- The size of signature $\Sigma$ is dominated by that of the Stern-like NIZKAoK $\Pi$, which is $\widetilde{\mathcal{O}}(|\xi| \cdot \log q) \cdot \omega(\log \lambda)$, where $|\xi|$ denotes the bit-size of the witnesstuple $\xi$. Overall, $\Sigma$ has bit-size $\widetilde{\mathcal{O}}(\lambda \cdot k)$.

Correctness. The correctness of the above scheme follows from the following facts: (i) the underlying argument system is perfectly complete; (ii) the underlying encryption scheme obtained by transforming the IBE scheme in [25] via CHK transformation [18] is correct.

Specifically, for an honest user, when he signs a message at time period $t$, he is able to demonstrate the possession of a valid tuple $\xi$ of the form (3). Therefore, with probability 1 , the resulting signature $\Pi$ will be accepted by the Verify algorithm, implied by the perfect completeness of the underlying argument system. As for the correctness of the Open algorithm, note that

$$
\begin{aligned}
\mathbf{c}_{2}-\mathbf{F}_{\text {ovk }}^{\top} \cdot \mathbf{c}_{1} & =\mathbf{G}^{\top} \cdot \mathbf{s}+\mathbf{e}_{2}+\left\lfloor\frac{q}{2}\right\rfloor \cdot \mathrm{id}-\mathbf{F}_{\text {ovk }}^{\top} \cdot\left(\mathbf{B}^{\top} \cdot \mathbf{s}+\mathbf{e}_{1}\right) \\
& =\left\lfloor\frac{q}{2}\right\rfloor \cdot \mathrm{id}+\mathbf{e}_{2}-\mathbf{F}_{\text {ovk }}^{\top} \cdot \mathbf{e}_{1}
\end{aligned}
$$


where $\left\|\mathbf{e}_{1}\right\| \leq B,\left\|\mathbf{e}_{2}\right\|_{\infty} \leq B$, and $\left\|\mathbf{f}_{i}\right\|_{\infty} \leq\left\lceil s_{\ell} \cdot \log m\right\rceil=\widetilde{\mathcal{O}}(\sqrt{n \cdot k})$, which is implied by Lemma 1. Recall that $q=\operatorname{poly}(n), m=\mathcal{O}(n \log q)$ and $B=\widetilde{\mathcal{O}}(\sqrt{n})$. Hence

$$
\left\|\mathbf{e}_{2}-\mathbf{F}_{\text {ovk }}^{\top} \cdot \mathbf{e}_{1}\right\|_{\infty} \leq B+m \cdot B \cdot \widetilde{\mathcal{O}}(\sqrt{n \cdot k})=\widetilde{\mathcal{O}}\left(n^{2}\right) .
$$

As long as we choose sufficiently large $q$, with probability 1 , the Open algorithm will recover id and correctness of the Open algorithm holds.

SECURITy. In Theorem 2, we prove that our scheme satisfies the security requirements put forward by Nakanishi et al. [51].

Theorem 2. In the random oracle model, the forward-secure group signature described in Section 3.1 satisfies full anonymity and forward-secure traceability requirements under the LWE and SIS assumptions.

The proof of the theorem is established by Lemma 6 and Lemma 7

Lemma 6. Suppose that one-time signature scheme $\mathcal{O T S}$ is strongly unforgeable. In the random oracle model, the forward-secure group signature scheme described in Section 3.1 is fully anonymous under the hardness of the $\mathrm{LWE}_{n, q, \chi}$ problem.

Proof. Denote $\mathcal{C}$ as the challenger and $\mathcal{A}$ as the adversary. Following [45], we prove this lemma using a series of computationally indistinguishable games. The first game Game 0 is the real experiment $\operatorname{Exp}_{\mathrm{FSGS}, \mathcal{A}}^{\text {Anon }}(\lambda, T, N)$ while the last game is such that the advantage of the adversary is 0 .

Game 0: In this game, $\mathcal{C}$ runs the experiment $\operatorname{Exp}_{\mathrm{FSGS}, \mathcal{A}}^{\text {Anon }}(\lambda, T, N)$ faithfully. In the challenge phase, $\mathcal{A}$ outputs a message $M^{*}$ together with two users $0 \leq i_{0}, i_{1} \leq N-1$ for the targeted time $t^{*}$. $\mathcal{C}$ responds by sending back a signature $\Sigma^{*}=\left(\right.$ ovk $\left.^{*}, \mathbf{c}_{1}^{*}, \mathbf{c}_{2}^{*}, \Pi^{*}, \operatorname{sig}^{*}\right) \leftarrow \operatorname{Sign}\left(\right.$ gpk, $\left.\mathbf{u s k}_{t^{*}}\left[i_{b}\right], t^{*}, M^{*}\right)$ for a random bit $b \in\{0,1\}$. Then the adversary outputs a bit $b^{\prime} \in\{0,1\}$ and this game returns 1 if $b^{\prime}=b$ and 0 otherwise. In this experiment, $\mathcal{C}$ replies with all random strings for oracles queries of $\mathcal{H}_{0}, \mathcal{H}_{1}$.

Game 1: In this game, we modify Game 0 in two aspects: (i) We generate the pair $\left(\right.$ ovk $^{*}$, osk $\left.^{*}\right)$ in the very beginning of the experiment; (ii) For the signature opening queries, if $\mathcal{A}$ asks for a valid signature of the form $\Sigma=$ (ovk, $\mathbf{c}_{1}, \mathbf{c}_{2}, \Pi$, sig) such that ovk $=$ ovk $^{*}$, then $\mathcal{C}$ outputs a random bit and aborts the experiment. Now we argue that the probability that $\mathcal{C}$ aborts is negligible and hence Game 0 and Game 1 are computationally indistinguishable. Actually, before the challenged signature is given to $\mathcal{A}$, ovk ${ }^{*}$ is independent of $\mathcal{A}^{\prime} s$ view, hence it is negligible that $\mathcal{A}$ queries a signature containing ovk* Furthermore, after the challenged signature is sent to $\mathcal{A}$, if $\mathcal{A}$ queries a new valid signature of the form (ovk ${ }^{*}, \mathbf{c}_{1}, \mathbf{c}_{2}, \Pi$, sig), then $\left(\left(\mathbf{c}_{1}, \mathbf{c}_{2}, \Pi\right)\right.$, sig $)$ is a successful forgery of the $\mathcal{O T S}$ scheme, which breaks the strong unforgeability of the $\mathcal{O} \mathcal{T S}$ scheme. This proves that $\mathcal{C}$ aborts with negligible probability. From now on, we assume that $\mathcal{A}$ will not query valid signature containing ovk*. 
Game 2: In this game, we change Game 1 in the following ways. First, instead of generating $\mathbf{B}$ using the TrapGen algorithm, we generate a uniformly random matrix $\mathbf{B}^{*}$ over $\mathbb{Z}_{q}^{n \times m}$. This change is indistinguishable to $\mathcal{A}$ since the matrix $\mathbf{B}$ is statistically close to uniform by Lemma 2 . Second, we program the random oracle $\mathcal{H}_{0}$ as follows. For query of ovk*, it generates a uniformly random matrix $\mathbf{G}^{*} \in \mathbb{Z}_{q}^{n \times \ell}$, let $\mathcal{H}_{0}\left(\mathrm{ovk}^{*}\right)=\mathbf{G}^{*}$, and return $\mathbf{G}^{*}$ to $\mathcal{A}$. This change also makes no difference to $\mathcal{A}$ since the output of $\mathcal{H}_{0}$ is uniformly random. For query of ovk $\neq$ ovk$^{*}$, we first sample $\mathbf{F}_{\text {ovk }} \leftarrow D_{\mathbb{Z}, s_{\ell}}^{m \times \ell}$, let $\mathcal{H}_{0}$ (ovk) $=\mathbf{G}=\mathbf{B}^{*} \cdot \mathbf{F}_{\text {ovk }} \bmod q$, and return $\mathbf{G}$ to $\mathcal{A}$. We then keep a record of (ovk, $\left.\mathbf{F}_{\text {ovk }}, \mathbf{G}\right)$. $\mathbf{G}$ generated in this way is statistically close to uniform by Lemma 1, Hence, this change does not affect $\mathcal{A}$ 's view non-negligibly. When $\mathcal{A}$ queries the opening oracle a signature (ovk, $\left.\mathbf{c}_{1}, \mathbf{c}_{2}, \Pi, \mathrm{sig}\right)$ with ovk $\neq \mathrm{ovk}^{*}$, $\mathcal{C}$ can use the recorded $\mathbf{F}_{\text {ovk }}$ to decrypt the ciphertext $\left(\mathbf{c}, \mathbf{c}_{2}\right)$. Hence $\mathcal{C}$ can answer all signature opening queries. It then follows that Game 2 and Game 1 are statistically indistinguishable.

Game 3: In this game,we modify Game 2 as follows. Instead of generating a real proof $\Pi^{*}$ for the challenged signature, we generate a simulated one without using the witness by programming the random oracle $\mathcal{H}_{1}$. Since our argument system is statistically zero-knowledge, the view of adversary $\mathcal{A}$ is statistically indistinguishable between Game 3 and Game 2.

Game 4: In this game, we change Game 3 as follows. Instead of computing

$$
\left(\mathbf{c}_{1}^{*}, \mathbf{c}_{2}^{*}\right)=\left(\mathbf{B}^{*^{\top}} \cdot \mathbf{s}+\mathbf{e}_{1}, \mathbf{G}^{*^{\top}} \cdot \mathbf{s}+\mathbf{e}_{2}+\left\lfloor\frac{q}{2}\right\rfloor \cdot \operatorname{Bin}\left(i_{b}\right)\right) \in \mathbb{Z}_{q}^{m} \times \mathbb{Z}_{q}^{\ell}
$$

in the challenged phase, where $\mathbf{s} \in \chi^{n}, \mathbf{e}_{1} \in \chi^{m}, \mathbf{e}_{2} \in \chi^{\ell}$, we let

$$
\left(\mathbf{c}_{1}^{*}, \mathbf{c}_{2}^{*}\right)=\left(\mathbf{z}_{1}^{*}, \mathbf{z}_{2}^{*}+\left\lfloor\frac{q}{2}\right\rfloor \cdot \operatorname{Bin}\left(i_{b}\right)\right),
$$

where $\mathbf{z}_{1}^{*}, \mathbf{z}_{2}^{*}$ are uniformly random vectors over $\mathbb{Z}_{q}^{m}$ and $\mathbb{Z}_{q}^{\ell}$. We claim that this modification is computationally indistinguishable to the view of the adversary $\mathcal{A}$ assuming the hardness of $\mathrm{LWE}_{n, q, \chi}$. Indeed, if we let $\mathbf{D}=$ $\left[\mathbf{B}^{*} \mid \mathbf{G}^{*}\right] \in \mathbb{Z}_{q}^{n \times(m+\ell)}, \mathbf{e}=\left(\mathbf{e}_{1} \| \mathbf{e}_{2}\right) \in \chi^{m+\ell}$, and $\mathbf{z}=\left(\mathbf{z}_{1}^{*} \| \mathbf{z}_{2}^{*}\right) \in \mathbb{Z}_{q}^{m+\ell}$, then to distinguish Game 3 and Game 4 is to distinguish $\left(\mathbf{D}, \mathbf{D}^{T} \cdot \mathbf{s}+\mathbf{e}\right)$ and $(\mathbf{D}, \mathbf{z})$. Recall that $\mathbf{B}^{*}$ and $\mathbf{G}^{*}$ are uniformly random matrices, so is $\mathbf{D}$.

Game 5: In this game, we slightly change Game 4 by substituting $\left(\mathbf{c}_{1}^{*}, \mathbf{c}_{2}^{*}\right)$ with a new independent and uniform tuple $\left(\mathbf{z}_{1}^{\prime}, \mathbf{z}_{2}^{\prime}\right)$. It is straightforward that Game 5 and Game 4 are statistically indistinguishable. Furthermore, the challenged signature in this game does not depends on the challenged bit $b$ any more, and hence the advantage of $\mathcal{A}$ in this game is 0 .

It then follows that $\operatorname{Adv}_{\mathrm{FSGS}, \mathcal{A}}^{\text {Anon }}(\lambda, T, N)$ is negligible in $\lambda$ because of the indistinguishability of the above games. This concludes the proof.

Lemma 7. In the random oracle model, the forward-secure group signature scheme described in Section 3.1 is forward-secure traceable under the hardness of the $\mathrm{SIS}_{n, \bar{m}, q, 2 \beta}^{\infty}$ problem, where $\bar{m}=(k+1) m$. 
Proof. Assume there is a PPT adversary $\mathcal{A}$ attacking the forward-secure traceability of the forward-secure group signature scheme with non-negligible probability, then we construct a new PPT adversary $\mathcal{B}$ attacking the $\operatorname{SIS}_{n, \bar{m}, q, 2 \beta}^{\infty}$ problem with non-negligible probability.

Given an SIS instance $\mathbf{C} \in \mathbb{Z}_{q}^{n \times \bar{m}}$, the goal of $\mathcal{B}$ is to find a non-zero vector $\mathbf{v} \in \mathbb{Z}_{q}^{\bar{m}}$ such that $\mathbf{C} \cdot \mathbf{v}=\mathbf{0} \bmod q$ and $\|\mathbf{v}\|_{\infty} \leq 2 \beta$. Towards this goal, $\mathcal{B}$ simulates the view of the adversary $\mathcal{A}$ attacking the forward-secure traceability. Initially, $\mathcal{B}$ lets $t=0$ and the set $\mathrm{CU}$ be empty and then generates group public key gpk, secret key for opening mosk, and some secret internal state as follows:

- Parse $\mathbf{C}$ as $\mathbf{C}=\left[\mathbf{C}_{0}\left|\mathbf{C}_{1}\right| \cdots \mid \mathbf{C}_{k}\right]$ for $\mathbf{C}_{j} \in \mathbb{Z}_{q}^{n \times m}, j \in\{0,1, \cdots, k\}$. It then generates the remaining public parameters as in Section 3.1

- Sample $\mathbf{z}=\left(\mathbf{z}_{0}\left\|\mathbf{z}_{1}\right\| \cdots \| \mathbf{z}_{k}\right) \in \mathbb{Z}^{\bar{m}}$, where each $\mathbf{z}_{i}$ is sampled from $D_{\mathbb{Z}^{m}, s_{k}}$. If $\|\mathbf{z}\|_{\infty}>\beta$, then repeat the sampling. Compute $\mathbf{u}=\mathbf{C} \cdot \mathbf{z} \bmod q$.

- Guess the targeted user $i^{*} \in\{0,1, \cdots, N-1\}$ and targeted forgery time period $t^{*} \in\{0,1, \cdots, T-1\}$ uniformly.

- Uncontrolled growth. Let id ${ }^{*}=\operatorname{Bin}\left(i^{*}\right)$ and $z^{*}=\operatorname{Bin}\left(t^{*}\right)$. Define $\mathbf{A}_{j}^{\mathrm{id}^{*}[j]}$ to be $\mathbf{C}_{j}$ for $j \in[\ell]$ and $\mathbf{A}_{\ell+j}^{z^{*}[j]}$ to be $\mathbf{C}_{\ell+j}$ for $j \in[d]$. Recall $k=\ell+d$.

- Controlled growth. Generate $\mathbf{A}_{j}^{1-\mathrm{id}{ }^{*}[j]}$ for all $j \in[\ell]$ via $\left(\mathbf{A}_{j}^{1-\mathrm{id}^{*}[j]}, \mathbf{S}_{j}\right) \leftarrow$ $\operatorname{TrapGen}(n, m, q)$ and $\mathbf{A}_{\ell+j}^{1-z^{*}[j]}$ for $j \in[d]$ via the algorithm $\left(\mathbf{A}_{\ell+j}^{1-z^{*}[j]}, \mathbf{S}_{\ell+j}\right) \leftarrow$ $\operatorname{TrapGen}(n, m, q)$.

- Generate a master key pair $(\mathbf{B}, \mathbf{S})$ via $\operatorname{TrapGen}(n, m, q)$.

$\mathcal{B}$ invokes $\mathcal{A}$ by sending gpk, mosk and then interacts with $\mathcal{A}$. At the start of each time period $t \in\{0,1, \cdots, T-1\}, \mathcal{B}$ announces the beginning of $t$ to $\mathcal{A}$. At current time period $t, \mathcal{B}$ responds to $\mathcal{A}$ 's queries as follows.

- When $\mathcal{A}$ queries the random oracles $\mathcal{H}_{0}, \mathcal{H}_{1}, \mathcal{B}$ replies with uniformly random strings and keeps a record of the queries.

- When $\mathcal{A}$ queries the secret key of member $i^{*}$, if $i^{*} \in \mathrm{CU}$ or $t \leq t^{*}, \mathcal{B}$ then abort: 2 . Otherwise, for each node $z \in \operatorname{Nodes}_{(t \rightarrow T-1)}$, it first computes the smallest index $d_{z, t}$ such that $1 \leq d_{z, t} \leq d$ and $z^{*}\left[d_{z, t}\right] \neq z\left[d_{z, t}\right]$. Then $\mathcal{B}$ computes $\mathbf{u s k}_{t}[i][z]$ via SampleD(ExtBasis $\left.\left(\mathbf{S}_{\ell+d_{z, t}}, \mathbf{A}_{\mathrm{id}^{*} \| z}\right), s_{k}\right)$ if $z$ is of length $d$ or via RandBasis(ExtBasis $\left.\left(\mathbf{S}_{\ell+d_{z, t}}, \mathbf{A}_{\mathrm{id}^{*} \| z}\right), s_{\ell+d_{z}}\right)$ if $z$ is of length $d_{z}<d$. Finally, $\mathcal{B}$ sets $\mathbf{u s k}_{t}[i]$ as in our construction and sends it to $\mathcal{A}$. Add $i^{*}$ to the set $\mathrm{CU}$.

- When $\mathcal{A}$ queries the secret key of member $i \neq i^{*}$, if $i \in \mathrm{CU}, \mathcal{B}$ then aborts. Otherwise, let id $=\operatorname{Bin}(i)$ and $\ell_{i}$ be the smallest index such that $1 \leq \ell_{i} \leq \ell$ and $\operatorname{id}\left[\ell_{i}\right] \neq \operatorname{id}^{*}\left[\ell_{i}\right]$. Compute usk $_{t}[i][z]$ via the algorithm SampleD(ExtBasis $\left.\left(\mathbf{S}_{\ell_{i}}, \mathbf{A}_{\mathrm{id} \| z}\right), \mathbf{u}, s_{k}\right)$ if $z$ is of length $d$ or via the algorithm RandBasis(ExtBasis $\left.\left(\mathbf{S}_{\ell_{i}}, \mathbf{A}_{\mathrm{id} \| z}\right), s_{\ell+d_{z}}\right)$ if $z$ is of length $d_{z}<d$ for $z \in \operatorname{Nodes}_{(t \rightarrow T-1)}$. Set $\mathbf{u s k}_{t}[i]$ as in our construction and send it to $\mathcal{A}$. Finally, add $i$ to the set CU.

2 This guarantees that once the secret key of member $i^{*}$ is queried, it is only queried at time $t$ such that $t>t^{*}$. 
- When $\mathcal{A}$ queries a signature on a message for user $i$. If $i \in \mathrm{CU}$ at current time $t, \mathcal{B}$ abort:3. Otherwise, if $i \neq i^{*}, \mathcal{B}$ responds as in our algorithm Sign using the corresponding witness. If $i=i^{*}, \mathcal{B}$ performs the same as in our algorithm Sign except that it generates a simulated proof $\Pi^{\prime}$ by programming the hash oracle $\mathcal{H}_{1}$ and returns $\Sigma=\left(\right.$ ovk, $\mathbf{c}_{1}, \mathbf{c}_{2}, \Pi^{\prime}$, sig) to $\mathcal{A}$.

It is worth noticing that the secret key of user $i^{*}$ is either never queried $\left(i^{*} \notin \mathrm{CU}\right)$ or is only queried at time $t$ with $t>t^{*}$.

We claim that $\mathcal{A}$ cannot distinguish whether it interacts with the real challenger or with $\mathcal{B}$. First, group public key gpk given to $\mathcal{A}$ is indistinguishable from the real one. This is because the output matrix $\mathbf{A}$ of the TrapGen algorithm is statistically close to uniform by Lemma 2 and $\mathbf{u}$ is statistically close to a uniform vector over $\mathbb{Z}_{q}^{n}$ by Lemma 1. Second, the secret signing key given to $\mathcal{A}$ is indistinguishable from the real one due to the fact that the outputs of RandBasis using two different bases are within negligible statistical distance by Lemma 5 . Third, the signature queries make no difference to the view of $\mathcal{A}$. This can be implied by the statistical zero-knowledge property of the underlying argument system.

When $\mathcal{A}$ halts and outputs a message $M^{*}$ and a signature $\Sigma^{*}$ at the targeted time period $t^{\prime}$ such that Verify $\left(\mathrm{gpk}, t^{\prime}, M^{*}, \Sigma^{*}\right)=1$ and $\Pi^{*}$ is not obtained by making a signing query at $M^{*}$, check $t^{\prime}=t^{*}$ holds or not. If not, indicating the guess of $t^{*}$ fails, then $\mathcal{B}$ aborts. Otherwise, $\mathcal{A}$ outputs $\left(t^{*}, M^{*}, \Sigma^{*}\right)$. Parse $\Sigma^{*}$ as

$$
\left(\mathrm{ovk}^{*}, \mathbf{c}_{1}^{*}, \mathbf{c}_{2}^{*},\left(\mathrm{CMT}_{i}^{*}\right)_{i=1}^{\kappa},\left(\mathrm{Ch}_{i}^{*}\right)_{i=1}^{\kappa},\left(\mathrm{RSP}_{i}^{*}\right)_{i=1}^{\kappa}, \operatorname{sig}^{*}\right) .
$$

Run id ${ }^{\prime} \leftarrow$ Open (gpk, mosk, $\left.t^{*}, M^{*}, \Sigma^{*}\right)$. If $\mathrm{id}^{\prime} \neq \mathrm{id}^{*}$, indicating that the guess of $i^{*}$ fails, then $\mathcal{B}$ aborts. Otherwise, $\mathcal{B}$ makes use of the forgery to solve the SIS problem as follows.

First, $\mathcal{A}$ must have queried $\mathcal{H}_{1}$ for the tuple $\left(M^{*},\left(\mathrm{CMT}_{i}^{*}\right)_{i=1}^{\kappa}, \mathbf{c}_{1}^{*}, \mathbf{c}_{2}^{*}, t^{*}\right)$, since the probability of guessing this value is at most $3^{-\kappa}$, which is negligible by our choice of $\kappa$. Let $\left(M^{*},\left(\mathrm{CMT}_{i}^{*}\right)_{i=1}^{\kappa}, \mathbf{c}_{1}^{*}, \mathbf{c}_{2}^{*}, t^{*}\right)$ be the $h$-th oracle query and $Q_{\mathcal{H}_{1}}$ be the total oracle queries $\mathcal{A}$ has made to $\mathcal{H}_{1}$. Next, $\mathcal{B}$ lets $h$ be the targeted forking point and replays $\mathcal{A}$ polynomial-number times. For each new run, $\mathcal{B}$ starts with the same random tape and random input as in the original run. Further, for the first $h-1$ queries of $\mathcal{H}_{1}, \mathcal{B}$ replies with the same random value as in the original run, but from $h$-th query on, $\mathcal{B}$ replies with fresh and independent value. Besides, for queries of $\mathcal{H}_{0}, \mathcal{B}$ always replies as in the original run.

Constructed in this way, $\left(M^{*},\left(\mathrm{CMT}_{i}^{*}\right)_{i=1}^{\kappa}, \mathbf{c}_{1}^{*}, \mathbf{c}_{2}^{*}, t^{*}\right)$ is always the $h$-th oracle query $\mathcal{A}$ made to $\mathcal{H}_{1}$. The improved forking lemma [15] implies that with probability $\geq \frac{1}{2}, \mathcal{B}$ obtains 3 -fork for the tuple $\left(M^{*},\left(\mathrm{CMT}_{i}^{*}\right)_{i=1}^{\kappa}, \mathbf{c}_{1}^{*}, \mathbf{c}_{2}^{*}, t^{*}\right)$ with pairwise distinct hash values $\mathrm{CH}_{h}^{(1)}, \mathrm{CH}_{h}^{(2)}, \mathrm{CH}_{h}^{(3)}$ and corresponding valid responses $\operatorname{RSP}_{h}^{(1)}, \operatorname{RSP}_{h}^{(2)}, \operatorname{RSP}_{h}^{(3)}$. A simple calculation shows that with probability $1-\left(\frac{7}{9}\right)^{\kappa}$, we have $\left\{\mathrm{CH}_{h, j}^{(1)}, \mathrm{CH}_{h, j}^{(2)}, \mathrm{CH}_{h, j}^{(3)}\right\}=\{1,2,3\}$ for some $j \in[\kappa]$. Therefore, $\operatorname{RSP}_{h, j}^{(1)}, \operatorname{RSP}_{h, j}^{(2)}$, $\operatorname{RSP}_{h, j}^{(3)}$ are 3 valid responses for all the challenges

\footnotetext{
${ }^{3}$ Note that $\mathcal{B}$ never answers the signing queries of corrupted users.
} 
$1,2,3$ w.r.t. the same commitment $\mathrm{CMT}_{j}^{*}$. Since COM is computationally binding, $\mathcal{B}$ is able to extract the witness tuple

$$
\xi^{*}=\left(\mathrm{id}, \mathbf{s}, \mathbf{e}_{1}, \mathbf{e}_{2}, \mathbf{v}_{\mathrm{id} \| z}\right)
$$

such that $\left\|\mathbf{v}_{\mathrm{id} \| z}\right\|_{\infty} \leq \beta,\|\mathbf{s}\|_{\infty} \leq B,\left\|\mathbf{e}_{1}\right\|_{\infty} \leq B,\left\|\mathbf{e}_{2}\right\|_{\infty} \leq B$ and

$$
\begin{gathered}
\mathbf{A}_{\mathrm{id} \| z} \cdot \mathbf{v}_{\mathrm{id} \| z}=\mathbf{u} \bmod q \\
\left(\mathbf{c}_{1}^{*}=\mathbf{B}^{T} \cdot \mathbf{s}+\mathbf{e}_{1}, \mathbf{c}_{2}^{*}=\left(\mathbf{G}^{*}\right)^{T} \cdot \mathbf{s}+\mathbf{e}_{2}+\left\lfloor\frac{q}{2}\right\rfloor \cdot \mathrm{id}\right) \in \mathbb{Z}_{q}^{m} \times \mathbb{Z}_{q}^{\ell},
\end{gathered}
$$

where $\mathbf{G}^{*}=\mathcal{H}_{0}\left(\right.$ ovk $\left.^{*}\right)$. Conditioned on guessing correctly $i^{*}, t^{*}$, we have $\mathrm{id}=\mathrm{id}^{*}$ and $z=z^{*}$. Therefore, $\mathbf{A}_{\mathrm{id} \| z}=\mathbf{C}$. Now we have $\mathbf{C} \cdot \mathbf{v}_{\mathrm{id} \| z}=\mathbf{u}=\mathbf{C} \cdot \mathbf{z} \bmod q$. We claim that $\mathbf{v}_{\mathrm{id} \| z} \neq \mathbf{z}$ with overwhelming probability. This is because $\mathcal{A}$ either queried the secret key of user id at time after $t^{*}$ or never queried the secret key at all (by successfully attacking forward-secure traceability), then $\mathbf{z}$ is not known to $\mathcal{A}$. Further, from the view of the adversary, $\mathbf{z}$ is from the distribution $D_{\Lambda^{\mathbf{u}}(\mathbf{C}), s_{k}}$ and hence has large min-entropy, which are implied by Lemma 1 . Therefore, $\mathbf{v}_{\mathrm{id} \| z} \neq \mathbf{z}$ with overwhelming probability. Hence $\mathbf{x}=\mathbf{v}_{\mathrm{id} \| z}-\mathbf{z} \neq \mathbf{0}$ and $\|\mathbf{x}\|_{\infty} \leq 2 \beta$. This implies that we solve the $\operatorname{SIS}_{n, \bar{m}, q, 2 \beta}^{\infty}$ problem with nonnegligible probability and hence our scheme is forward-secure traceable.

\section{The Underlying Zero-Knowledge Argument System}

In Section 4.1, we recall the extension, decomposition, and permutation techniques from 4433. Then we describe in Section 4.2 our statistical ZKAoK protocol that will be used in generating group signatures.

\subsection{Extension, Decomposition, and Permutation}

Extensions. For $\mathfrak{m} \in \mathbb{Z}$, let $B_{3 \mathfrak{m}}$ be the set of all vectors in $\{-1,0,1\}^{3 \mathfrak{m}}$ having exactly $\mathfrak{m}$ coordinates $-1, \mathfrak{m}$ coordinates 1 , and $\mathfrak{m}$ coordinates 0 and $\mathcal{S}_{\mathfrak{m}}$ be the set of all permutations on $\mathfrak{m}$ elements. Let $\oplus$ be the addition operation modulo 2 . Define the following functions

$-\operatorname{ext}_{3}:\{-1,0,1\}^{\mathfrak{m}} \rightarrow \mathrm{B}_{3 \mathfrak{m}}$ that transforms a vector $\mathbf{v}=\left(v_{1}, \ldots, v_{\mathfrak{m}}\right)^{\top}$ to vector $\left(\mathbf{v}\left\|(-\mathbf{1})^{\mathfrak{m}-n_{-1}}\right\| \mathbf{0}^{\mathfrak{m}-n_{0}} \| \mathbf{1}^{\mathfrak{m}-n_{1}}\right)^{\top}$, where $n_{j}$ is the number of element $j$ in the vector $\mathbf{v}$ for $j \in\{-1,0,1\}$.

- enc $2:\{0,1\}^{\mathfrak{m}} \rightarrow\{0,1\}^{2 \mathfrak{m}}$ that transforms a vector $\mathbf{v}=\left(v_{1}, \ldots, v_{\mathfrak{m}}\right)^{\top}$ to vector $\left(v_{1}, 1-v_{1}, \ldots, v_{\mathfrak{m}}, 1-v_{\mathfrak{m}}\right)^{\top}$.

Decompositions And Permutations. We now recall the integer decomposition technique. For any $B \in \mathbb{Z}^{+}$, define $p_{B}=\lfloor\log B\rfloor+1$ and the sequence $B_{1}, \ldots, B_{p_{B}}$ as $B_{j}=\left\lfloor\frac{B+2^{j-1}}{2^{j}}\right\rfloor$ for each $j \in\left[p_{B}\right]$. As observed in [44], it satis-

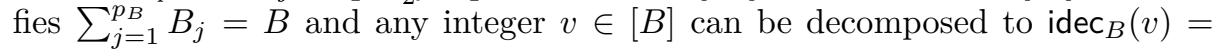
$\left(v^{(1)}, \ldots, v^{\left(p_{B}\right)}\right)^{\top} \in\{0,1\}^{p_{B}}$ such that $\sum_{j=1}^{p_{B}} B_{j} \cdot v^{(j)}=v$. This decomposition procedure is described in a deterministic manner as follows: 
1. $v^{\prime}:=v$

2. For $j=1$ to $p_{B}$ do:

(i) If $v^{\prime} \geq B_{j}$ then $v^{(j)}:=1$, else $v^{(j)}:=0$;

(ii) $v^{\prime}:=v^{\prime}-B_{j} \cdot v^{(j)}$.

3. Output $\operatorname{idec}_{B}(v)=\left(v^{(1)}, \ldots, v^{\left(p_{B}\right)}\right)^{\top}$.

Next, for any positive integers $\mathfrak{m}, B$, we define the function $\operatorname{vdec}_{\mathfrak{m}, B}$ that transforms a vector $\mathbf{w}=\left(w_{1}, \ldots, w_{\mathfrak{m}}\right)^{\top} \in[-B, B]^{\mathfrak{m}}$ to a vector of the following form:

$$
\mathbf{w}^{\prime}=\left(\sigma\left(w_{1}\right) \cdot \operatorname{idec}_{B}\left(\left|w_{1}\right|\right)\|\cdots\| \sigma\left(w_{\mathfrak{m}}\right) \cdot \operatorname{idec}_{B}\left(\left|w_{\mathfrak{m}}\right|\right)\right) \in\{-1,0,1\}^{\mathfrak{m} p_{B}},
$$

where $\forall j \in[\mathfrak{m}]: \sigma\left(w_{j}\right)=0$ if $w_{j}=0 ; \sigma\left(w_{j}\right)=-1$ if $w_{j}<0 ; \sigma\left(w_{j}\right)=1$ if $w_{j}>0$.

Define the matrix $\mathbf{H}_{\mathfrak{m}, B}=\left[\begin{array}{ccc}B_{1}, \ldots, B_{p_{B}} & & \\ & \ddots & \\ & & B_{1}, \ldots, B_{p_{B}}\end{array}\right] \in \mathbb{Z}^{\mathfrak{m} \times \mathfrak{m} p_{B}}$ and

its extension $\widehat{\mathbf{H}}_{\mathfrak{m}, B}=\left[\mathbf{H}_{\mathfrak{m}, B} \mid \mathbf{0}^{\mathfrak{m} \times 2 \mathfrak{m} p_{B}}\right] \in \mathbb{Z}^{\mathfrak{m} \times 3 \mathfrak{m} p_{B}}$. Let $\widehat{\mathbf{w}}=\operatorname{ext}_{3}\left(\mathbf{w}^{\prime}\right) \in \mathrm{B}_{3 \mathfrak{m} p_{B}}$, then one can see that $\widehat{\mathbf{H}}_{\mathfrak{m}, B} \cdot \widehat{\mathbf{w}}=\mathbf{w}$ and for any $\psi \in \mathcal{S}_{3 \mathfrak{m} p_{B}}$, the following equivalence holds:

$$
\widehat{\mathbf{w}} \in \mathrm{B}_{3 \mathfrak{m} p_{B}} \Leftrightarrow \psi(\widehat{\mathbf{w}}) \in \mathrm{B}_{3 \mathfrak{m} p_{B}} .
$$

Define the following permutation.

- For any $\mathbf{e}=\left(e_{1}, \ldots, e_{\mathfrak{m}}\right)^{\top} \in\{0,1\}^{\mathfrak{m}}$, define $\Pi_{\mathbf{e}}: \mathbb{Z}^{2 \mathfrak{m}} \rightarrow \mathbb{Z}^{2 \mathfrak{m}}$ that maps a vector $\mathbf{v}=\left(v_{1}^{0}, v_{1}^{1}, \ldots, v_{\mathfrak{m}}^{0}, v_{\mathfrak{m}}^{1}\right)^{\top}$ to $\left(v_{1}^{e_{1}}, v_{1}^{1-e_{1}}, \ldots, v_{\mathfrak{m}}^{e_{\mathfrak{m}}}, v_{\mathfrak{m}}^{1-e_{\mathfrak{m}}}\right)^{\top}$.

One can see that, for any $\mathbf{z}, \mathbf{e} \in\{0,1\}^{\mathfrak{m}}$, the following equivalence holds:

$$
\mathbf{v}=\operatorname{enc}_{2}(\mathbf{z}) \Leftrightarrow \Pi_{\mathbf{e}}(\mathbf{v})=\operatorname{enc}_{2}(\mathbf{z} \oplus \mathbf{e}) .
$$

\subsection{The Underlying Zero-Knowledge Argument System}

We now describe a statistical ZKAoK that will be invoked by the signer when generating group signatures. The protocol is developed from Stern-like techniques proposed by Ling et al. [44] and Langlois et al. [33].

Public input $\gamma: \mathbf{A}_{0} \in \mathbb{Z}_{q}^{n \times m}, \mathbf{A}_{j}^{b} \in \mathbb{Z}_{q}^{n \times m}$ for $(b, j) \in\{0,1\} \times[k], \mathbf{u} \in \mathbb{Z}_{q}^{n}$, $\mathbf{B} \in \mathbb{Z}_{q}^{n \times m}, \mathbf{G} \in \mathbb{Z}_{q}^{n \times \ell},\left(\mathbf{c}_{1}, \mathbf{c}_{2}\right) \in \mathbb{Z}_{q}^{m} \times \mathbb{Z}_{q}^{\ell}, t \in\{0,1, \cdots, T-1\}$.

Secret input $\xi$ : id $\in\{0,1\}^{\ell}, \mathbf{s} \in \chi^{n}, \mathbf{e}_{1} \in \chi^{m}, \mathbf{e}_{2} \in \chi^{\ell}, \mathbf{v}_{\mathrm{id} \| z} \in \mathbb{Z}^{(\ell+d+1) m}$ with $z=\operatorname{Bin}(t)$.

\section{Prover's goal:}

$$
\left\{\begin{array}{l}
\mathbf{A}_{\mathrm{id} \| z} \cdot \mathbf{v}_{\mathrm{id} \| z}=\mathbf{u} \bmod q,\left\|\mathbf{v}_{\mathrm{id} \|_{z}}\right\|_{\infty} \leq \beta \\
\mathbf{c}_{1}=\mathbf{B}^{\top} \cdot \mathbf{s}+\mathbf{e}_{1} \bmod q, \quad \mathbf{c}_{2}=\mathbf{G}^{\top} \cdot \mathbf{s}+\mathbf{e}_{2}+\left\lfloor\frac{q}{2}\right\rfloor \cdot \operatorname{id} \bmod q \\
\|\mathbf{s}\|_{\infty} \leq B,\left\|\mathbf{e}_{1}\right\|_{\infty} \leq B,\left\|\mathbf{e}_{2}\right\|_{\infty} \leq B
\end{array}\right.
$$


We first rearrange the above conditions. Let $\mathbf{A}^{\prime}=\left[\mathbf{A}\left|\mathbf{A}_{1}^{0}\right| \mathbf{A}_{1}^{1}|\cdots| \mathbf{A}_{\ell}^{0} \mid \mathbf{A}_{\ell}^{1}\right] \in$ $\mathbb{Z}_{q}^{(2 \ell+1) m}, \mathbf{A}_{\mathrm{id}}=\left[\mathbf{A}_{0}\left|\mathbf{A}_{1}^{\mathrm{id}[1]}\right| \cdots \mid \mathbf{A}_{1}^{\mathrm{id}[\ell]}\right] \in \mathbb{Z}_{q}^{(\ell+1) m}$ and $\left.\mathbf{A}^{\prime \prime}=\mathbf{A}_{\ell+1}^{z[1]}|\cdots| \mathbf{A}_{\ell+1}^{z[d]}\right] \in$ $\mathbb{Z}_{q}^{d m}$. Then $\mathbf{A}_{\text {id } \| z}=\left[\mathbf{A}_{\text {id }} \mid \mathbf{A}^{\prime \prime}\right] \in \mathbb{Z}_{q}^{(\ell+d+1) m}$. Let $\mathbf{v}_{\text {id }}=\left(\mathbf{v}_{0}\left\|\mathbf{v}_{1}\right\| \cdots \| \mathbf{v}_{\ell}\right), \mathbf{w}_{2}=$ $\left(\mathbf{v}_{\ell+1}\|\cdots\| \mathbf{v}_{\ell+d}\right)$ with each $\mathbf{v}_{i} \in \mathbb{Z}^{m}$. Then $\mathbf{v}_{\text {id } \| z}=\left(\mathbf{v}_{\text {id }} \| \mathbf{w}_{2}\right)$. Therefore $\mathbf{A}_{\text {id } \| z}$. $\mathbf{v}_{\mathrm{id} \| z}=\mathbf{u} \bmod q$ is equivalent to

$$
\mathbf{A}_{\mathrm{id}} \cdot \mathbf{v}_{\mathrm{id}}+\mathbf{A}^{\prime \prime} \cdot \mathbf{w}_{2}=\mathbf{u} \bmod q .
$$

Since id is part of secret input, $\mathbf{A}_{\mathrm{id}}$ should not be explicitly given. We note that Langlois et al. [33] already addressed this problem. The idea is as follows: they first added $\ell$ suitable zero-blocks of size $m$ to vector $\mathbf{v}_{i d}$ and then obtained the extended vector $\mathbf{w}_{1}=\left(\mathbf{v}_{0}\left\|\mathbf{v}_{1}^{0}\right\| \mathbf{v}_{1}^{1}\|\cdots\| \mathbf{v}_{\ell}^{0} \| \mathbf{v}_{\ell}^{1}\right) \in \mathbb{Z}^{(2 \ell+1) m}$, where the added zero-blocks are $\mathbf{v}_{1}^{1-\mathrm{id}[1]}, \ldots, \mathbf{v}_{\ell}^{1-\mathrm{id}[\ell]}$ and $\mathbf{v}_{i}^{\mathrm{id}[i]}=\mathbf{v}_{i}, \forall i \in[\ell]$. Now one can check that equation (7) is equivalent to

$$
\mathbf{A}^{\prime} \cdot \mathbf{w}_{1}+\mathbf{A}^{\prime \prime} \cdot \mathbf{w}_{2}=\mathbf{u} \bmod q .
$$

Let $\mathbf{B}^{\prime}=\left[\begin{array}{ccc}\mathbf{B}^{\top} & \mathbf{I}_{m} & \mathbf{0}^{m \times \ell} \\ \mathbf{G}^{\top} & \mathbf{0}^{\ell \times m} & \mathbf{I}_{\ell}\end{array}\right], \mathbf{B}^{\prime \prime}=\left[\begin{array}{c}\mathbf{0}^{m \times \ell} \\ \lfloor q / 2\rfloor \mathbf{I}_{\ell}\end{array}\right]$, and $\mathbf{w}_{3}=\left(\mathbf{s}\left\|\mathbf{e}_{1}\right\| \mathbf{e}_{2}\right) \in$ $\mathbb{Z}^{n+m+\ell}$. Then one can check that $\mathbf{c}_{1}=\mathbf{B}^{\top} \cdot \mathbf{s}+\mathbf{e}_{1} \bmod q, \mathbf{c}_{2}=\mathbf{G}^{\top} \cdot \mathbf{s}+$ $\mathbf{e}_{2}+\left\lfloor\frac{q}{2}\right\rfloor \cdot \operatorname{id} \bmod q$ is equivalent to

$$
\mathbf{B}^{\prime} \cdot \mathbf{w}_{3}+\mathbf{B}^{\prime \prime} \cdot \mathrm{id}=\left(\mathbf{c}_{1} \| \mathbf{c}_{2}\right) \bmod q .
$$

Using basic algebra, we can transform equations (8) and (9) into one equation of the following form:

$$
\mathbf{M}_{0} \cdot \mathbf{w}_{0}=\mathbf{u}_{0} \bmod q,
$$

where $\mathbf{M}_{0}, \mathbf{u}_{0}$ are built from $\mathbf{A}^{\prime}, \mathbf{A}^{\prime \prime}, \mathbf{B}^{\prime}, \mathbf{B}^{\prime \prime}$ and $\mathbf{u},\left(\mathbf{c}_{1} \| \mathbf{c}_{2}\right)$, respectively, and $\mathbf{w}_{0}=\left(\mathbf{w}_{1}\left\|\mathbf{w}_{2}\right\| \mathbf{w}_{3} \| \mathrm{id}\right)$.

Now we can use the decomposition and extension techniques described in Section 4.1 to handle our secret vectors. Let $L_{1}=3(2 \ell+1) m p_{\beta}, L_{2}=3 d m p_{\beta}$, $L_{3}=3(n+m+\ell) p_{B}$, and $L=L_{1}+L_{2}+L_{3}+2 \ell$. We transform our secret vector $\mathbf{w}_{0}$ to vector $\mathbf{w}=\left(\widehat{\mathbf{w}}_{1}\left\|\widehat{\mathbf{w}}_{2}\right\| \widehat{\mathbf{w}}_{3} \| \widehat{\mathrm{id}}\right) \in\{-1,0,1\}^{L}$ of the following form:

$-\widehat{\mathbf{w}}_{1}=\left(\widehat{\mathbf{v}}_{0}\left\|\widehat{\mathbf{v}}_{1}^{0}\right\| \widehat{\mathbf{v}}_{1}^{1}\|\cdots\| \widehat{\mathbf{v}}_{\ell}^{0} \| \widehat{\mathbf{v}}_{\ell}^{1}\right) \in\{-1,0,1\}^{L_{1}}$ with $\widehat{\mathbf{v}}_{0}=\operatorname{ext}_{3}\left(\operatorname{vdec}_{m, \beta}\left(\mathbf{v}_{0}\right)\right) \in$ $\mathrm{B}_{3 m p_{\beta}}, \forall i \in[\ell], \widehat{\mathbf{v}}_{i}^{1-\operatorname{id}[i]}=\mathbf{0}^{3 m p_{\beta}}$ and $\widehat{\mathbf{v}}_{i}^{\operatorname{id}[i]}=\operatorname{ext}_{3}\left(\operatorname{vdec}_{m, \beta}\left(\mathbf{v}_{i}^{\operatorname{id}[i]}\right)\right) \in \mathrm{B}_{3 m p_{\beta}}$;

$-\widehat{\mathbf{w}}_{2}=\operatorname{ext}_{3}\left(\operatorname{vdec}_{d m, \beta}\left(\mathbf{w}_{2}\right)\right) \in \mathrm{B}_{3 d m p_{\beta}} ;$

$-\widehat{\mathbf{w}}_{3}=\operatorname{ext}_{3}\left(\operatorname{vdec}_{n+m+\ell, B}\left(\mathbf{w}_{3}\right)\right) \in \mathrm{B}_{3(n+m+\ell) p_{B}} ;$

$-\widehat{\mathrm{id}}=\mathrm{enc}_{2}(\mathrm{id}) \in\{0,1\}^{2 \ell}$.

Using basic algebra, we can form public matrix $\mathbf{M}$ such that

$$
\mathbf{M} \cdot \mathbf{w}=\mathbf{M}_{0} \cdot \mathbf{w}_{0}=\mathbf{u}_{0} \bmod q .
$$

Up to this point, we have transformed the considered relations into equation of the desired form $\mathbf{M} \cdot \mathbf{w}=\mathbf{u} \bmod q$. We now specify the set VALID that 
contains the secret vector $\mathbf{w}$, the set $\mathcal{S}$ and permutations $\left\{\Gamma_{\phi}: \phi \in \mathcal{S}\right\}$ such that the conditions in (1) hold.

Define VALID to be the set of vectors of the form $\mathbf{z}=\left(\mathbf{z}_{1}\left\|\mathbf{z}_{2}\right\| \mathbf{z}_{3} \| \mathbf{z}_{4}\right) \in$ $\{-1,0,1\}^{L}$ such that there exists $\mathbf{x} \in\{0,1\}^{\ell}$

$-\mathbf{z}_{1}=\left(\mathbf{y}_{0}\left\|\mathbf{y}_{1}^{0}\right\| \mathbf{y}_{1}^{1}\|\cdots\| \mathbf{y}_{\ell}^{0} \| \mathbf{y}_{\ell}^{1}\right) \in\{-1,0,1\}^{3(2 \ell+1) m p_{\beta}}$ with $\mathbf{y}_{0} \in \mathrm{B}_{3 m p_{\beta}}$ and for each $i \in[\ell], \mathbf{y}_{i}^{1-\mathbf{x}[i]}=\mathbf{0}^{3 m p_{\beta}}, \mathbf{y}_{i}^{\mathbf{x}}{ }^{[i]} \in \mathrm{B}_{3 m p_{\beta}}$;

$-\mathbf{z}_{2} \in \mathrm{B}_{3 d m p_{\beta}}$ and $\mathbf{z}_{3} \in \mathrm{B}_{3(n+m+\ell) p_{B}}$;

$-\mathbf{z}_{4}=\operatorname{enc}_{2}(\mathbf{x}) \in\{0,1\}^{2 \ell}$.

Clearly, our vector $\mathbf{w}$ belongs to the tailored set VALID.

Now, let $\mathcal{S}=\left(\mathcal{S}_{3 m p_{\beta}}\right)^{2 \ell+1} \times \mathcal{S}_{3 d m p_{\beta}} \times \mathcal{S}_{3(n+m+\ell) p_{B}} \times\{0,1\}^{\ell}$. For any

$$
\phi=\left(\psi_{0}, \psi_{1}^{0}, \psi_{1}^{1}, \ldots, \psi_{\ell}^{0}, \psi_{\ell}^{1}, \eta_{2}, \eta_{3}, \mathbf{e}\right) \in \mathcal{S}, \mathbf{e}=\left(e_{1}, \ldots, e_{\ell}\right)^{\top},
$$

define the permutation $\Gamma_{\phi}: \mathbb{Z}^{L} \rightarrow \mathbb{Z}^{L}$ as follows. When applied to a vector

$$
\mathbf{z}=\left(\mathbf{y}_{0}\left\|\mathbf{y}_{1}^{0}\right\| \mathbf{y}_{1}^{1}\|\cdots\| \mathbf{y}_{\ell}^{0}\left\|\mathbf{y}_{\ell}^{1}\right\| \mathbf{z}_{2}\left\|\mathbf{z}_{3}\right\| \mathbf{z}_{4}\right) \in \mathbb{Z}^{L}
$$

where the first $2 \ell+1$ blocks are of size $3 m p_{\beta}$ and the last three blocks are of size $3 d m p_{\beta}, 3(n+m+\ell) p_{B}$ and $2 \ell$, respectively; it transforms $\mathbf{z}$ to vector $\Gamma_{\phi}(\mathbf{z})$ of the following form:

$$
\begin{aligned}
& \left(\psi\left(\mathbf{y}_{0}\right)\left\|\psi_{1}^{e_{1}}\left(\mathbf{y}_{1}^{e_{1}}\right)\right\| \psi_{1}^{1-e_{1}}\left(\mathbf{y}_{1}^{1-e_{1}}\right)\|\cdots\| \psi_{\ell}^{e_{\ell}}\left(\mathbf{y}_{\ell}^{e_{\ell}}\right)\left\|\psi_{\ell}^{1-e_{\ell}}\left(\mathbf{y}_{\ell}^{1-e_{\ell}}\right)\right\|\right. \\
& \left.\eta_{2}\left(\mathbf{z}_{2}\right)\left\|\eta_{3}\left(\mathbf{z}_{3}\right)\right\| \Pi_{\mathbf{e}}\left(\mathbf{z}_{4}\right)\right) .
\end{aligned}
$$

Based on the equivalences observed in (4) and (5), it can be checked that if $\mathbf{z} \in$ VALID for some $\mathbf{x} \in\{0,1\}^{\ell}$, then $\Gamma_{\phi}(\mathbf{z}) \in$ VALID for some $\mathbf{x} \oplus \mathbf{e} \in\{0,1\}^{\ell}$. In other words, the conditions in (11) hold, and therefore, we can obtain the desired statistical ZKAoK protocol.

\section{Acknowledgements}

The research is supported by Singapore Ministry of Education under Research Grant MOE2016-T2-2-014(S). Khoa Nguyen is also supported by the Gopalakrishnan - NTU Presidential Postdoctoral Fellowship 2018.

\section{References}

1. M. Abdalla and L. Reyzin. A New Forward-Secure Digital Signature Scheme. In ASIACRYPT 2000, volume 1976 of LNCS, pages 116-129. Springer, 2000.

2. M. Ajtai. Generating Hard Instances of Lattice Problems (Extended Abstract). In STOC 1996, pages 99-108. ACM, 1996.

3. J. Alwen and C. Peikert. Generating Shorter Bases for Hard Random Lattices. In STACS 2009, pages 75-86, 2009. 
4. R. Anderson. Two Remarks on Public Key Cryptology. Technical report, University of Cambridge, Computer Laboratory, 2002.

5. B. Applebaum, D. Cash, C. Peikert, and A. Sahai. Fast cryptographic primitives and circular-secure encryption based on hard learning problems. In CRYPTO 2009, volume 5677 of $L N C S$, pages 595-618. Springer, 2009.

6. M. Bellare, D. Micciancio, and B. Warinschi. Foundations of Group Signatures: Formal Definitions, Simplified Requirements, and a Construction Based on General Assumptions. In EUROCRYPT 2003, volume 2656 of LNCS, pages 614-629. Springer, 2003.

7. M. Bellare and S. K. Miner. A Forward-Secure Digital Signature Scheme. In CRYPTO 1999, volume 1666 of $L N C S$, pages 431-448. Springer, 1999.

8. M. Bellare, H. Shi, and C. Zhang. Foundations of Group Signatures: The Case of Dynamic Groups. In CT-RSA 2005, volume 2656 of LNCS. Springer, 2005.

9. D. Boneh, X. Boyen, and E.-J. Goh. Hierarchical Identity based Encryption with Constant Size Ciphertext. In EUROCRYPT 2005, volume 3494 of $L N C S$, pages 440-456. Springer, 2005.

10. D. Boneh and H. Shacham. Group Signatures with Verifier-Local Revocation. In ACM-CCS 2004, pages 168-177. ACM, 2004.

11. C. Boschini, J. Camenisch, and G. Neven. Floppy-sized group signatures from lattices. In ACNS 2018, volume 10892 of LNCS, pages 163-182. Springer, 2018.

12. X. Boyen. Lattice mixing and vanishing trapdoors: A framework for fully secure short signatures and more. In PKC 2010, volume 6056 of LNCS, pages 499-517. Springer, 2010.

13. X. Boyen, H. Shacham, E. Shen, and B. Waters. Forward-Secure Signatures with Untrusted Update. In ACM-CCS 2006, pages 191-200. ACM, 2006.

14. Z. Brakerski, C. Gentry, and V. Vaikuntanathan. (leveled) fully homomorphic encryption without bootstrapping. In ITCS 2012, pages 309-325. ACM, 2012.

15. E. Brickell, D. Pointcheval, S. Vaudenay, and M. Yung. Design Validations for Discrete Logarithm Based Signature Schemes. In PKC 2000, pages 276-292. Springer, 2000 .

16. J. Camenisch, G. Neven, and M. Rückert. Fully Anonymous Attribute Tokens from Lattices. In $S C N$ 2012, volume 7485 of $L N C S$, pages 57-75. Springer, 2012.

17. R. Canetti, S. Halevi, and J. Katz. A Forward-Secure Public-Key Encryption Scheme. In EUROCRYPT 2003, volume 2656 of LNCS, pages 255-271. Springer, 2003.

18. R. Canetti, S. Halevi, and J. Katz. Chosen-Ciphertext Security from IdentityBased Encryption. In EUROCRYPT 2004, volume 3027 of LNCS, pages 207-222. Springer, 2004.

19. D. Cash, D. Hofheinz, E. Kiltz, and C. Peikert. Bonsai Trees, or How to Delegate a Lattice Basis. In EUROCRYPT 2010, volume 6110 of $L N C S$, pages 523-552. Springer, 2010.

20. D. Chaum and E. van Heyst. Group Signatures. In EUROCRYPT 1991, volume 547 of $L N C S$, pages 257-265. Springer, 1991.

21. S. Cheng, K. Nguyen, and H. Wang. Policy-Based Signature Scheme from Lattices. Des. Codes Cryptography, 81(1):43-74, 2016.

22. R. del Pino, V. Lyubashevsky, and G. Seiler. Lattice-based group signatures and zero-knowledge proofs of automorphism stability. In ACM-CCS 2018, pages 574591. ACM, 2018.

23. Y. Dodis, J. Katz, S. Xu, and M. Yung. Key-Insulated Public Key Cryptosystems. In EUROCRYPT 2002, volume 2332 of $L N C S$, pages 65-82. Springer, 2002. 
24. A. Fiat and A. Shamir. How to Prove Yourself: Practical Solutions to Identification and Signature Problems. In CRYPTO 1986, volume 263 of LNCS, pages 186-194. Springer, 1987.

25. C. Gentry, C. Peikert, and V. Vaikuntanathan. How to Use a Short Basis: Trapdoors for Hard Lattices and New Cryptographic Constructions. In STOC 2008, pages 197-206. ACM, 2008.

26. S. D. Gordon, J. Katz, and V. Vaikuntanathan. A Group Signature Scheme from Lattice Assumptions. In ASIACRYPT 2010, volume 6477 of LNCS, pages 395-412. Springer, 2010.

27. G. Itkis and L. Reyzin. Forward-Secure Signatures with Optimal Signing and Verifying. In CRYPTO 2001, volume 2139 of LNCS, pages 332-354. Springer, 2001.

28. M. Kansal, R. Dutta, and S. Mukhopadhyay. Forward Secure Efficient Group Signature in Dynamic Setting using Lattices. IACR Cryptology ePrint Archive, 2017:1128. Available at: https://eprint.iacr.org/2017/1128

29. A. Kawachi, K. Tanaka, and K. Xagawa. Concurrently Secure Identification Schemes Based on the Worst-Case Hardness of Lattice Problems. In ASIACRYPT 2008, volume 5350 of $L N C S$, pages 372-389. Springer, 2008.

30. A. Kiayias, Y. Tsiounis, and M. Yung. Traceable signatures. In EUROCRYPT 2004, volume 3027 of $L N C S$, pages 571-589. Springer, 2004.

31. A. Kiayias and M. Yung. Secure Scalable Group Signature with Dynamic Joins and Separable authorities. IJSN, 1(1):24-45, 2006.

32. F. Laguillaumie, A. Langlois, B. Libert, and D. Stehlé. Lattice-Based Group Signatures with Logarithmic Signature Size. In ASIACRYPT 2013, volume 8270 of LNCS, pages 41-61. Springer, 2013.

33. A. Langlois, S. Ling, K. Nguyen, and H. Wang. Lattice-Based Group Signature Scheme with Verifier-Local Revocation. In $P K C$ 2014, volume 8383 of LNCS, pages 345-361. Springer, 2014. Corrected full version: http://eprint.iacr.org/2014/033.

34. B. Libert, S. Ling, F. Mouhartem, K. Nguyen, and H. Wang. Signature Schemes with Efficient Protocols and Dynamic Group Signatures from Lattice Assumptions. In ASIACRYPT 2016, volume 10032 of LNCS, pages 373-403. Springer, 2016.

35. B. Libert, S. Ling, F. Mouhartem, K. Nguyen, and H. Wang. Zero-Knowledge Arguments for Matrix-Vector Relations and Lattice-Based Group Encryption. In ASIACRYPT 2016, volume 10032 of LNCS, pages 101-131, 2016.

36. B. Libert, S. Ling, F. Mouhartem, K. Nguyen, and H. Wang. Adaptive Oblivious Transfer with Access Control from Lattice Assumptions. In ASIACRYPT 2017, volume 10624 of $L N C S$, pages 533-563. Springer, 2017.

37. B. Libert, S. Ling, K. Nguyen, and H. Wang. Zero-Knowledge Arguments for Lattice-Based Accumulators: Logarithmic-Size Ring Signatures and Group Signatures Without Trapdoors. In EUROCRYPT 2016, volume 9666 of LNCS, pages 1-31. Springer, 2016.

38. B. Libert, S. Ling, K. Nguyen, and H. Wang. Zero-knowledge arguments for latticebased prfs and applications to e-cash. In ASIACRYPT 201\%, volume 10626 of LNCS, pages 304-335. Springer, 2017.

39. B. Libert, S. Ling, K. Nguyen, and H. Wang. Lattice-based zero-knowledge arguments for integer relations. In CRYPTO 2018, volume 10992 of $L N C S$, pages 700-732. Springer, 2018.

40. B. Libert, F. Mouhartem, and K. Nguyen. A Lattice-Based Group Signature Scheme with Message-Dependent Opening. In ACNS 2016, volume 9696 of $L N C S$, pages 137-155. Springer, 2016. 
41. B. Libert, J.-J. Quisquater, and M. Yung. Forward-Secure Signatures in Untrusted Update Environments: Efficient and Generic Constructions. In ACM-CCS 200\%, pages 266-275. ACM, 2007.

42. B. Libert, J.-J. Quisquater, and M. Yung. Key Evolution Systems in Untrusted Update Environments. ACM Transactions on Information and System Security (TISSEC), 13(4):37, 2010.

43. B. Libert and M. Yung. Dynamic Fully Forward-Secure Group Signatures. In Asia-CCS 2010, pages 70-81. ACM, 2010.

44. S. Ling, K. Nguyen, D. Stehlé, and H. Wang. Improved Zero-Knowledge Proofs of Knowledge for the ISIS Problem, and Applications. In PKC 2013, volume 7778, pages 107-124. Springer, 2013.

45. S. Ling, K. Nguyen, and H. Wang. Group Signatures from Lattices: Simpler, Tighter, Shorter, Ring-Based. In PKC 2015, volume 9020 of LNCS, pages 427449. Springer, 2015.

46. S. Ling, K. Nguyen, H. Wang, and Y. Xu. Lattice-Based Group Signatures: Achieving Full Dynamicity with Ease. In ACNS 2017, volume 10355 of LNCS, pages 293-312. Springer, 2017.

47. S. Ling, K. Nguyen, H. Wang, and Y. Xu. Accountable tracing signatures from lattices. IACR Cryptology ePrint Archive, 2018:1251, 2018. To appear at CT-RSA 2019.

48. S. Ling, K. Nguyen, H. Wang, and Y. Xu. Constant-size group signatures from lattices. In $P K C$ 2018, volume 10770 of $L N C S$, pages 58-88. Springer, 2018.

49. D. Micciancio and C. Peikert. Hardness of SIS and LWE with Small Parameters. In CRYPTO 2013, volume 8042 of $L N C S$, pages 21-39. Springer, 2013.

50. D. Micciancio and O. Regev. Worst-Case to Average-Case Reductions Based on Gaussian Measures. SIAM Journal on Computing, 37(1):267-302, 2007.

51. T. Nakanishi, Y. Hira, and N. Funabiki. Forward-Secure Group Signatures from Pairings. In Pairing 2009, pages 171-186. Springer, 2009.

52. K. Nguyen, B. H. M. Tan, and H. Wang. Zero-knowledge password policy check from lattices. In $I S C$ 2017, volume 10599 of $L N C S$, pages 92-113. Springer, 2017.

53. P. Q. Nguyen, J. Zhang, and Z. Zhang. Simpler Efficient Group Signatures from Lattices. In PKC 2015, volume 9020 of $L N C S$, pages 401-426. Springer, 2015.

54. C. Peikert and A. Rosen. Efficient Collision-Resistant Hashing from Worst-Case Assumptions on Cyclic Lattices. In TCC 2006, volume 3876 of LNCS, pages 145166. Springer, 2006.

55. O. Regev. On Lattices, Learning with Errors, Random Linear Codes, and Cryptography. In STOC 2005, pages 84-93. ACM, 2005.

56. Y. Sakai, K. Emura, G. Hanaoka, Y. Kawai, T. Matsuda, and K. Omote. Group Signatures with Message-Dependent Opening. In Pairing 2012, volume 7708 of LNCS, pages 270-294. Springer, 2012.

57. D. X. Song. Practical Forward Secure Group Signature Schemes. In ACM-CCS 2001, pages 225-234. ACM, 2001.

58. J. Stern. A New Paradigm for Public Key Identification. IEEE Transactions on Information Theory, 42(6):1757-1768, 1996. 


\section{A Some Remarks on [28] (ePrint 2017/1128)}

In [28, Kansal, Dutta, and Mukhopadhyay proposed a forward-secure group signature scheme from lattices in the model of Libert and Yung [43. Unfortunately, it can be observed that their proposed scheme does not satisfy the correctness and security requirements.

The version of the scheme posted on 27-Nov-2017 15:26:21 UTC contains the following shortcomings.

- The scheme does not satisfy the correctness requirement. The opening algorithm, on input a signature generated by user $i$, does not output $i$. In fact, the transcript for user $i$ stored by the group manager is transcript ${ }_{i}=$ $\left(\mathbf{v}_{i}^{(0)}, i, \mathbf{u v k}[i], \operatorname{sig}_{i},\left[t_{1}, t_{2}\right]\right)$. However, when signing messages at time $t_{1}+j$ with $0<j \leq t_{2}-t_{1}$, the user $i$ encrypts $\mathbf{v}_{i}^{(j)}$, which is never seen by the group manager and which is unrelated to $\mathbf{v}_{i}^{(0)}$. Hence, the decryption procedure can only recovers $\mathbf{v}_{i}^{(j)}$ and no user is being traced in this case. (Readers are referred to Page 22 (Join algorithm) and Page 25 (Sign algorithm) in 28. for more details.)

- The scheme does not satisfy the anonymity requirement. The signature generated by user $i$ at time period $t_{j}$ contains matrix $\mathbf{C}_{i}^{(j)}$, which is part of the updated certificate and which should be kept secret. Therefore, two signatures generated by the same user at the same period can easily be linked. (Readers are referred to Page 24 (Update algorithm) and Page 27 (equation (13)) in 28 for more details.)

We note that in an updated version of the scheme, posted on 18-Jan-2018 17:33:37 UTC, the signature does not contain matrix $\mathbf{C}_{i}^{(j)}$, but the validity of the signature now cannot be publicly verified. That is because, in order to verify the underlying zero-knowledge argument system of Section 5 (Page 27), one needs to be given matrix $\mathbf{C}_{\mathrm{id}_{i}}^{(j)}$ that encodes the secret identity of the signer and that is not publicly known. In other words, this updated scheme also does not work.

We further observe that, a version of the scheme posted on 19-Apr-2018 07:26:41 UTC still contains the following shortcoming.

- The signatures generated by malicious users cannot be properly identified. In fact, to tackle with the first problem we have put forwarded above, the authors allow all users to update their transcripts to contain $\mathbf{v}_{i}^{(j)}$ at time $t_{j}$. However, this enables all malicious user to update the transcript arbitrarily. Therefore, employing the transcript to identify the signer when opening signatures is meaningless. (Readers are referred to the updating algorithm in Page 25.) 